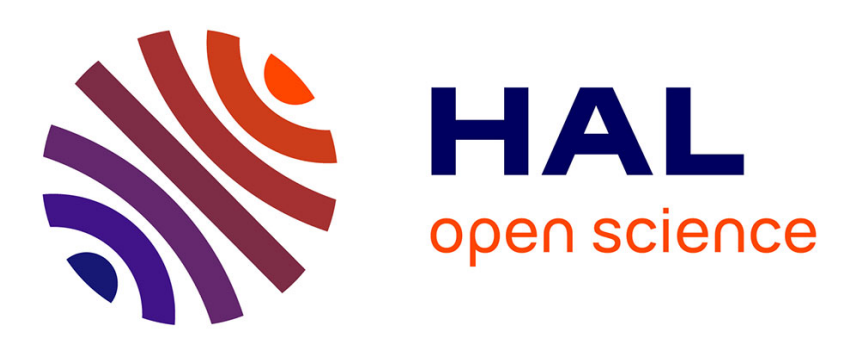

\title{
Decoupled time-marching schemes in computational cardiac electrophysiology and ECG numerical simulation
}

Miguel Angel Fernández, Nejib Zemzemi

\section{To cite this version:}

Miguel Angel Fernández, Nejib Zemzemi. Decoupled time-marching schemes in computational cardiac electrophysiology and ECG numerical simulation. Mathematical Biosciences, 2010, 226 (1), pp.58-75. 10.1016/j.mbs.2010.04.003 . inria-00411510v3

\section{HAL Id: inria-00411510 https://hal.inria.fr/inria-00411510v3}

Submitted on 7 Apr 2010

HAL is a multi-disciplinary open access archive for the deposit and dissemination of scientific research documents, whether they are published or not. The documents may come from teaching and research institutions in France or abroad, or from public or private research centers.
L'archive ouverte pluridisciplinaire HAL, est destinée au dépôt et à la diffusion de documents scientifiques de niveau recherche, publiés ou non, émanant des établissements d'enseignement et de recherche français ou étrangers, des laboratoires publics ou privés. 
INSTITUT NATIONAL DE RECHERCHE EN INFORMATIQUE ET EN AUTOMATIQUE

Decoupled time-marching schemes in computational cardiac electrophysiology and ECG numerical simulation

Miguel A. Fernández — Nejib Zemzemi

\section{$\mathbf{N}^{\circ} \mathbf{7 0 2 2}$}

August 2009

Thème BIO

\section{apport}

derecherche 



\title{
RIN RIA
}

\section{Decoupled time-marching schemes in computational cardiac electrophysiology and ECG numerical simulation}

\author{
Miguel A. Fernándę* Nejib Zemzemi许 \\ Thème BIO - Systèmes biologiques \\ Projet REO \\ Rapport de recherche $\mathrm{n}^{\circ} 7022$ - August 2009 - 38 pages
}

\begin{abstract}
This work considers the approximation of the cardiac bidomain equations, either isolated or coupled with the torso, via first order semi-implicit time-marching schemes involving a fully decoupled computation of the unknown fields (ionic state, transmembrane potential, extracellular and torso potentials). For the isolated bidomain system, we show that the Gauss-Seidel and Jacobi like splittings do not compromise energy stability; they simply alter the energy norm. Within the framework of the numerical simulation of electrocardiograms (ECG), these bidomain splittings are combined with an explicit Robin-Robin treatment of the heart-torso coupling conditions. We show that the resulting schemes allow a fully decoupled (energy) stable computation of the heart and torso fields, under an additional hyperbolic-CFL like condition. The accuracy and convergence rate of the considered schemes are investigated numerically with a series of numerical experiments.
\end{abstract}

Key-words: Cardiac electrophysiology, forward problem, electrocardiogram, bidomain equations, heart-torso coupling, time discretization, explicit coupling, finite element method, Robin transmission conditions, stability analysis.

\footnotetext{
* INRIA, REO project-team

† Université Paris 11, Laboratoire de mathématiques d'Orsay, F-91405 Orsay Cedex,
} France

Unité de recherche INRIA Rocquencourt

Domaine de Voluceau, Rocquencourt, BP 105, 78153 Le Chesnay Cedex (France)

Téléphone : +33139635511 - Télécopie : +331396353 30 


\section{Schémas de marche en temps découplés en éléctrophysiologie cardiaque computationnelle et simulation numérique de l'ECG}

Résumé : Ce travail aborde l'appproximation des équations bidomaine, isolées ou couplées avec le thorax, par des schémas semi-implicites de premier ordre, permettant un calcul découplé des inconnues (état ionique, potentiel transmembranaire, potentiel extracellulaire et potentiel thoracique). Pour les équations bidomaine isolées, nous montrons que les découplages de type Gauss-Seidel et Jacobi ne compromettent pas la stabilité (au sens de l'énergie), ils simplement modifient la norme de l'énergie. Dans le cadre de la simulation numérique de l'électrocardiogramme (ECG), nous proposons de combiner ces techniques avec un traitement Robin-Robin explicite du couplage cœur-thorax. Les schémas ainsi obtenus permettent un calcul complètement découplé et stable (sous une simple condition CFL-hyperbolique) des champs cardiaques et thoraciques. La précision et taux de convergence des schémas proposés sont illustrés par des expériences numériques.

Mots-clés : Électrophysiologie cardiaque, problème direct, électrocardiogramme, équation bidomaine, couplage cœur-thorax, discrétisation en temps, couplage explicite, méthode des éléments finis, conditions de Robin, analyse de stabilité. 


\section{Introduction}

Computational models of cardiac electrophysiology typically incorporate the cell membrane activity and the intra- and extracellular components of cardiac tissue by means of the bidomain model (see e.g. 44, 40). This mathematical model can be formulated as a three-field system (ionic state, transmembrane and extracellular potentials) coupling a non-linear reaction-diffusion equation, an elliptic equation and a non-linear system of ODE (alternative formulations are discussed in [25, 36]).

The rapid dynamics of the ODE system, acting on the reaction terms, lead to the presence of a sharp propagating wavefront, which often requires fine resolutions in space and in time. As a result, fully implicit time-marching is extremely difficult to perform since it involves the resolution of a large system of non-linear equations at each time step (see e.g. [25, 8, 34]). Attempts to reduce this computational complexity (without compromising too much numerical stability) consist in introducing some sort of explicit treatment within the time-marching procedure. For instance, by considering semi-implicit (see e.g. [45, 31, 14, 2, 5, 19]) or operator splitting (see e.g. [27, 46, 50]) schemes. All these approaches uncouple the ODE system (ionic state and non-linear reaction terms) from the electro-diffusive components (transmembrane and extracellular potentials). However, only a few works 45, 31, 2, 50 propose a decoupled (Gauss-Seidel like) time-marching of the three fields.

In this paper we go further in the investigation of this kind of decoupling techniques, by providing a general energy based stability analysis that covers both the Gauss-Seidel and the Jacobi like approaches. In particular, we show that these electro-diffusive splittings do not compromise the stability of the resulting scheme. They simply alter the energy norm and time step restrictions are uniquely dictated by the semi-implicit treatment of the ODE system and the non-linear reaction terms. We also illustrate numerically that the splitting preserves the first-order time accuracy of the original electro-diffusive coupling.

In the second part, we propose to extend these time-marching techniques to the numerical simulation of the electrocardiogram (ECG), namely, the forward problem of cardiac electrophysiology (see e.g. 30]). The bidomain equations have then to be coupled to a generalized Laplace equation, describing the electrical potential within the surrounding torso tissue. The heart-torso coupling is enforced through standard interface conditions, ensuring a perfect electrical balance (see e.g. 28, 40, 44). This results in a coupled four-field problem (ionic state, transmembrane, extracellular and torso potentials) coupling a nonlinear system of ODEs, a non-linear reaction-diffusion equation and two elliptic equations (see e.g. [30, 44]).

Traditionally, the heart-torso coupling has been treated using two different approaches (see e.g. 30]). The so called heart-torso uncoupling approximation (see e.g. [37, 39, 30, 4]) uncouples the heart and torso problems by neglecting the torso-to-heart electrical feedback (i.e. the heart is isolated). Although this approach is very appealing in terms of computational cost, it can compromise the accuracy of the corresponding ECG signals (see e.g. [30, 40, 4]). The second approach, the so called heart-torso full coupling, treats the heart-torso interface conditions in a fully implicit fashion and, therefore, requires the resolution of a large heart-torso system at each time step (see e.g. [45, 31, 40, 4]). To the best of our knowledge, none of the current approaches is able to provide accurate ECG 
signals (i.e. close to heart-torso full coupling) with a decoupled computation of the extracellular and torso potentials.

In this paper, we introduce a series of time-marching schemes for ECG numerical simulation involving a fully decoupled computation of the ionic state, the transmembrane potential, the extracellular potential and the torso potential.

The main idea consists in combining the above mentioned Gauss-Seidel or Jacobi like bidomain spplitings, with an explicit Robin-Robin treatment (derived from [1]) of the heart-torso coupling. Note that, since the time discretization of the two (quasi-static) elliptic equations does not produce numerical dissipation, conventional explicit Dirichlet-Neumann heart-torso coupling might lead to numerical instability. An energy based stability analysis shows that the proposed schemes are stable under and additional mild hyperbolic-CFL like condition. Optimal convergence rate, on the contrary, calls for a more stringent condition between the space and time discretization parameters.

The remainder of this paper is organized as follows. In the next section we briefly recall the coupled system of equations describing the electrical activity of the heart and its interaction with the surrounding torso tissue. The time discretization of the bidomain equations is addressed in section $\$ 3$ using GaussSeidel and Jacobi like ecletrodiffusive splittings. The stability of the resulting schemes is analyzed by means of energy arguments. Section $\$ 4$ is devoted to the discretization of the heart-torso system. The schemes analyzed in section $\$ 3$ are then combined with a specific explicit Robin-Robin treatment of the heart-torso coupling. The energy based stability of the resulting schemes is investigated. Numerical evidence of the stability and accuracy is provided in section \$5. with a mono-dimensional test problem and a three-dimensional study based on realistic heart and torso geometries. A summary of the results and some concluding remarks are given in section $\$ 6$.

\section{Mathematical models}

This section contains standard material (see e.g. [4, Chapter 2]). We introduce the notation and the coupled system of equations commonly used to model the electrical activity of the heart and its interaction with surrounding tissue (extramyocardial regions).

\subsection{Isolated heart}

The bidomain equations, originally derived in [48, are the most widely accepted mathematical model of the macroscopic electrical activity of the heart (see e.g. the monographs [4, 40]). This model is usually formulated in terms of three variables: the transmembrane potential $V_{\mathrm{m}}$, the extracellular potential $u_{\mathrm{e}}$ and the ionic state $w$ (possibly vector valued). These space and time dependent variables are defined in $\Omega_{\mathrm{H}} \times(0, T)$, where $\Omega_{\mathrm{H}}$ and $(0, T)$ denote, respectively, the heart domain and the time interval of interest.

The governing equations consist of a coupled system of ODE, a nonlinear reaction-diffusion equation and an elliptic equation, with appropriate boundary 
and initial conditions (see e.g. [44, 40]):

$$
\begin{aligned}
& \partial_{t} w+g\left(V_{\mathrm{m}}, w\right)=0, \quad \text { in } \quad \Omega_{\mathrm{H}} \times(0, T), \\
& \chi_{\mathrm{m}} \partial_{t} V_{\mathrm{m}}+I_{\mathrm{ion}}\left(V_{\mathrm{m}}, w\right)-\operatorname{div}\left(\boldsymbol{\sigma}_{\mathrm{i}} \boldsymbol{\nabla} V_{\mathrm{m}}\right)-\operatorname{div}\left(\boldsymbol{\sigma}_{\mathrm{i}} \boldsymbol{\nabla} u_{\mathrm{e}}\right)=I_{\text {app }}, \quad \text { in } \quad \Omega_{\mathrm{H}} \times(0, T) \text {, } \\
& -\operatorname{div}\left(\left(\boldsymbol{\sigma}_{\mathrm{i}}+\boldsymbol{\sigma}_{\mathrm{e}}\right) \boldsymbol{\nabla} u_{\mathrm{e}}\right)-\operatorname{div}\left(\boldsymbol{\sigma}_{\mathrm{i}} \boldsymbol{\nabla} V_{\mathrm{m}}\right)=0, \quad \text { in } \quad \Omega_{\mathrm{H}} \times(0, T), \\
& \boldsymbol{\sigma}_{\mathrm{i}} \boldsymbol{\nabla} V_{\mathrm{m}} \cdot \boldsymbol{n}+\boldsymbol{\sigma}_{\mathrm{i}} \boldsymbol{\nabla} u_{\mathrm{e}} \cdot \boldsymbol{n}=0, \quad \text { on } \quad \Sigma \times(0, T), \\
& \boldsymbol{\sigma}_{\mathrm{e}} \boldsymbol{\nabla} u_{\mathrm{e}} \cdot \boldsymbol{n}=0, \quad \text { on } \quad \Sigma \times(0, T), \\
& V_{\mathrm{m}}(\boldsymbol{x}, 0)=V_{\mathrm{m}}^{0}(\boldsymbol{x}), \quad w(\boldsymbol{x}, 0)=w^{0}(\boldsymbol{x}), \quad \forall \boldsymbol{x} \in \Omega_{\mathrm{H}} .
\end{aligned}
$$

Here, $\chi_{\mathrm{m}} \stackrel{\text { def }}{=} A_{\mathrm{m}} C_{\mathrm{m}}$ where $A_{\mathrm{m}}$ is a geometrical quantity, $C_{\mathrm{m}}$ denotes the membrane capacitance and tensors $\boldsymbol{\sigma}_{\mathrm{i}}$ and $\boldsymbol{\sigma}_{\mathrm{e}}$ represent, respectively, the intra- and extracellular conductivities. The term $I_{\mathrm{ion}}\left(V_{\mathrm{m}}, w\right) \stackrel{\text { def }}{=} A_{\mathrm{m}} i_{\text {ion }}\left(V_{\mathrm{m}}, w\right)$ denotes the ionic current across the membrane and $I_{\text {app }}$ a given external current stimulus. The explicit expression of functions $g$ and $i_{\text {ion }}$ depends on the considered cell ionic model (see e.g. [44, 40, and the references therein). At last, $\boldsymbol{n}$ stands for the outward unit normal to $\Sigma \stackrel{\text { def }}{=} \partial \Omega_{\mathrm{H}}$ (see Figure 1 ), and $V_{\mathrm{m}}^{0}, w^{0}$ are given initial data.

The boundary conditions (2.4)-2.5) state that the intra- and extracellular currents do not propagate outside the heart. While (2.4) is a widely accepted condition (see e.g. [48, 28, 40, 44]), the enforcement of (2.5) is only justified under an isolated heart assumption (see [4, 40, ). The coupled system of equations (2.1)-(2.6) is often known in the literature as isolated bidomain model (see e.g. [14, 15, 44]). The interested reader is referred to [16, 3, 7, 49] for the mathematical analysis of problem (2.1)-(2.6).

The choice of the formulation (2.1)-(2.6) is motivated by the decoupling timemarching schemes introduced in section $\$ 3$, Other formulations of the bidomain equations and their impact on the performance of the algebraic solvers have been discussed in [25, 36]. A recent review of numerical methods for the bidomain equations can be found in 32 .

Remark 2.1 The complexity of (2.1)-(2.6) can be reduced by using, instead of (2.2) and (2.4), the so-called monodomain approximation:

$$
\begin{aligned}
\chi_{\mathrm{m}} \partial_{t} V_{\mathrm{m}}+I_{\mathrm{ion}}\left(V_{\mathrm{m}}, w\right)-\operatorname{div}\left(\boldsymbol{\sigma} \nabla V_{\mathrm{m}}\right)=I_{\text {app }}, & \text { in } \quad \Omega_{\mathrm{H}}, \\
\boldsymbol{\sigma} \nabla V_{\mathrm{m}} \cdot \boldsymbol{n}=0, & \text { on } \quad \Sigma,
\end{aligned}
$$

where $\boldsymbol{\sigma} \stackrel{\text { def }}{=} \sigma_{\mathrm{i}}\left(\boldsymbol{\sigma}_{\mathrm{i}}+\boldsymbol{\sigma}_{\mathrm{e}}\right)^{-1} \boldsymbol{\sigma}_{\mathrm{e}}$ is the bulk conductivity tensor (see e.g. [29, 13, 15, 381). Note that (2.7) decouples de computation of $V_{\mathrm{m}}$ from that of $u_{\mathrm{e}}$. Under the isolating condition (2.5), 2.7) can be interpreted as the zeroth-order approximation of (2.2) and (2.4) with respect to a parameter, $0 \leq \epsilon<1$, which measures the gap between the anisotropy ratios of the intra- and extracellular domains (see [13, 15] for details). Although several simulation analysis (see 
e.g. [13, 38]) suggest that the monodomain approximation may be adequate for some propagation studies in isolated hearts, it cannot be applied in all situations since it neglects the extracellular feedback into $V_{\mathrm{m}}$ (see e.g. [17, 13, 38] and Remark 2.3 below).

\subsection{Coupling with torso: ECG modeling}

The myocardium is surrounded by a volume conductor, $\Omega_{\mathrm{T}}$, which contains all the extramyocardial regions (see Figure 1). As a matter of fact, ECG signals monitor the electrical activity of the heart from potential measurements at the torso skin surface $\Gamma_{\text {ext }}$. The torso volume is commonly modeled as a passive conductor (generalized Laplace equation), electrically coupled to the heart across the heart-torso interface $\Sigma$. The resulting coupled system can be formulated in

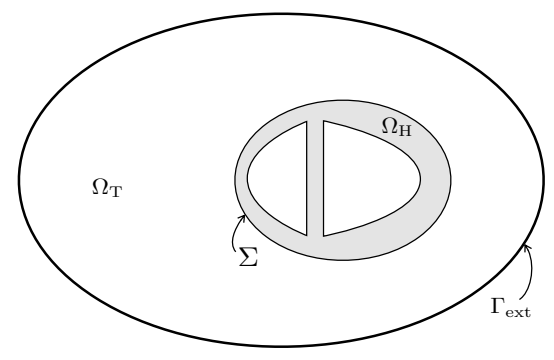

Figure 1: Two-dimensional geometrical description: heart domain $\Omega_{\mathrm{H}}$, torso domain $\Omega_{\mathrm{T}}$ (extramyocardial regions), heart-torso interface $\Sigma$ and torso external boundary $\Gamma_{\text {ext }}$.

terms of $V_{\mathrm{m}}, u_{\mathrm{e}}, w$ and the torso potential $u_{\mathrm{T}}$, as follows (see e.g. [44, 40]):

$$
\begin{aligned}
& \partial_{t} w+g\left(V_{\mathrm{m}}, w\right)=0, \quad \text { in } \quad \Omega_{\mathrm{H}} \times(0, T), \\
& \chi_{\mathrm{m}} \partial_{t} V_{\mathrm{m}}+I_{\text {ion }}\left(V_{\mathrm{m}}, w\right)-\operatorname{div}\left(\boldsymbol{\sigma}_{\mathrm{i}} \boldsymbol{\nabla} V_{\mathrm{m}}\right)-\operatorname{div}\left(\boldsymbol{\sigma}_{\mathrm{i}} \boldsymbol{\nabla} u_{\mathrm{e}}\right)=I_{\mathrm{app}}, \quad \text { in } \quad \Omega_{\mathrm{H}} \times(0, T), \\
& -\operatorname{div}\left(\left(\boldsymbol{\sigma}_{\mathrm{i}}+\boldsymbol{\sigma}_{\mathrm{e}}\right) \boldsymbol{\nabla} u_{\mathrm{e}}\right)-\operatorname{div}\left(\boldsymbol{\sigma}_{\mathrm{i}} \boldsymbol{\nabla} V_{\mathrm{m}}\right)=0, \quad \text { in } \quad \Omega_{\mathrm{H}} \times(0, T), \\
& -\operatorname{div}\left(\boldsymbol{\sigma}_{\mathrm{T}} \boldsymbol{\nabla} u_{\mathrm{T}}\right)=0, \quad \text { in } \Omega_{\mathrm{T}} \times(0, T), \\
& \boldsymbol{\sigma}_{\mathrm{T}} \boldsymbol{\nabla} u_{\mathrm{T}} \cdot \boldsymbol{n}_{\mathrm{T}}=0, \quad \text { on } \quad \Gamma_{\mathrm{ext}} \times(0, T), \\
& \boldsymbol{\sigma}_{\mathrm{i}} \boldsymbol{\nabla} V_{\mathrm{m}} \cdot \boldsymbol{n}+\boldsymbol{\sigma}_{\mathrm{i}} \boldsymbol{\nabla} u_{\mathrm{e}} \cdot \boldsymbol{n}=0, \quad \text { on } \quad \Sigma \times(0, T), \\
& u_{\mathrm{T}}=u_{\mathrm{e}}, \quad \text { on } \quad \Sigma \times(0, T), \\
& \boldsymbol{\sigma}_{\mathrm{e}} \boldsymbol{\nabla} u_{\mathrm{e}} \cdot \boldsymbol{n}=-\boldsymbol{\sigma}_{\mathrm{T}} \boldsymbol{\nabla} u_{\mathrm{T}} \cdot \boldsymbol{n}_{\mathrm{T}}, \quad \text { on } \quad \Sigma \times(0, T), \\
& V_{\mathrm{m}}(\boldsymbol{x}, 0)=V_{\mathrm{m}}^{0}(\boldsymbol{x}), \quad w(\boldsymbol{x}, 0)=w^{0}(\boldsymbol{x}), \quad \forall \boldsymbol{x} \in \Omega_{\mathrm{H}}
\end{aligned}
$$


Here, $\boldsymbol{\sigma}_{\mathrm{T}}$ stands for the conductivity tensor of the torso tissue and $\boldsymbol{n}_{\mathrm{T}}$ for the outward unit normal to the external boundary $\Gamma_{\text {ext }} \stackrel{\text { def }}{=} \partial \Omega_{\mathrm{T}} \backslash \Sigma$ (see Figure 1 ).

The boundary condition 2.12 states that no current can flow from the external torso surface $\Gamma_{\text {ext }}$, whereas (2.14)-2.15) enforce a perfect electric balance between the heart and torso domains (see e.g. [48, 28, 40, 44]).

The coupled system of equations $2.8-2.16$ is often known in the literature as full bidomain or coupled bidomain model (see e.g. [13, 44]). It can be considered as the state-of-the-art in the modeling of the ECG or, equivalently, the forward problem of cardiac electrophysiology (see e.g. [30, 40, 44]). The interested reader is referred to 6 for the mathematical analysis of problem (2.8)-(2.16), and to [30] (see also [4]) for a review of the numerical methods.

Remark 2.2 A common approach to reduce the computational complexity of (2.8)-2.16) consists in uncoupling the computation of $\left(w, V_{\mathrm{m}}, u_{\mathrm{e}}\right)$ and $u_{\mathrm{T}}$, by neglecting the electrical torso-to-heart feedback (see e.g. [13, 37, 30]). Thus, the coupling condition 2.15 is replaced by

$$
\boldsymbol{\sigma}_{\mathrm{e}} \boldsymbol{\nabla} u_{\mathrm{e}} \cdot \boldsymbol{n}=0, \quad \text { on } \quad \Sigma,
$$

which amounts to work with an isolated bidomain model, as described in the previous subsection. Thereafter, the torso potential $u_{\mathrm{T}}$ is recovered by solving (2.11) with boundary conditions (2.12)-(2.14). Despite this approach is very appealing in terms of computational cost, numerical evidence has shown that it can compromise the accuracy of the ECG signals (see e.g. [30, 40, 4] and the numerical study of subsection \$5.2.3). The heart-torso uncoupling approximation is often further simplified by replacing the interface condition (2.14) by a (multi-) dipole representation of the cardiac source (see e.g. [23, [26]).

Remark 2.3 The monodomain approximation (2.7) can be combined with the heart-torso uncoupling framework of Remark 2.2 (see e.g. [26, 37, 4]). This yields a simplified mathematical model which allows a fully decoupled computation of $V_{\mathrm{m}}, u_{\mathrm{e}}$ and $u_{\mathrm{T}}$. However, as noticed in [4. Section 5.2] (see also [13]), without the uncoupling assumption 2.17) the monodomain approximation becomes

$$
\begin{array}{r}
\chi_{\mathrm{m}} \partial_{t} V_{\mathrm{m}}+I_{\mathrm{ion}}\left(V_{\mathrm{m}}, w\right)-\operatorname{div}\left(\boldsymbol{\sigma} \nabla V_{\mathrm{m}}\right)=I_{\mathrm{app}}, \quad \text { in } \quad \Omega_{\mathrm{H}}, \\
\boldsymbol{\sigma} \boldsymbol{\nabla} V_{\mathrm{m}} \cdot \boldsymbol{n}=-\mu \boldsymbol{\sigma}_{\mathrm{e}} \boldsymbol{\nabla} u_{\mathrm{e}} \cdot \boldsymbol{n}, \quad \text { on } \quad \Sigma,
\end{array}
$$

where $0<\mu<1$ is a dimensionless parameter related to the local conductivities. Note that in (2.18) $V_{\mathrm{m}}$ and $u_{\mathrm{e}}$ are still coupled. Therefore, under the full hearttorso coupling 2.14-2.15, the monodomain approximation does not reduce the computational complexity with respect to (2.8)-(2.16).

\section{Decoupled time-marching for the bidomain eq- uation}

In this section we analyze some time-discretization schemes for the isolated bidomain system (2.1)-(2.6). The main feature of the analyzed schemes is that they all allow a decoupled (Gauss-Seidel or Jacobi like) computation of $V_{\mathrm{m}}$ and $u_{\mathrm{e}}$, without compromising stability. 


\subsection{Preliminaries}

In what follows, we will consider the usual Lebesgue and Sobolev spaces, $L^{m}(\Omega)$ and $H^{m}(\Omega)$ respectively $(m>0)$, for a domain $\Omega \subset \mathbb{R}^{3}$. Then, for a given $X \subset \partial \Omega$ (with meas $(X)>0$ ), we define $H_{X}^{1}(\Omega)$ as the subspace of $H^{1}(\Omega)$ with vanishing trace on $X$. The $L^{2}(\Omega)$-norm is denoted by $\|\cdot\|_{0, \Omega}$ and the vanishing mean value subspace of $L^{2}(\Omega)$ by $L_{0}^{2}(\Omega)$.

Problem 2.1]-2.6) can be cast into weak form as follows (see e.g. 6]): for $t>0$, find $w(\cdot, t) \in L^{\infty}\left(\Omega_{\mathrm{H}}\right), V_{\mathrm{m}}(\cdot, t) \in H^{1}\left(\Omega_{\mathrm{H}}\right)$ and $u_{\mathrm{e}}(\cdot, t) \in H^{1}\left(\Omega_{\mathrm{H}}\right) \cap L_{0}^{2}\left(\Omega_{\mathrm{H}}\right)$, such that

$$
\begin{gathered}
\int_{\Omega_{\mathrm{H}}}\left(\partial_{t} w+g\left(V_{\mathrm{m}}, w\right)\right) \xi \mathrm{d} \boldsymbol{x}=0, \\
\chi_{\mathrm{m}} \int_{\Omega_{\mathrm{H}}}\left(\partial_{t} V_{\mathrm{m}}+I_{\mathrm{ion}}\left(V_{\mathrm{m}}, w\right)\right) \phi \mathrm{d} \boldsymbol{x}+\int_{\Omega_{\mathrm{H}}} \boldsymbol{\sigma}_{\mathrm{i}} \boldsymbol{\nabla}\left(V_{\mathrm{m}}+u_{\mathrm{e}}\right) \cdot \boldsymbol{\nabla} \phi \mathrm{d} \boldsymbol{x}=\int_{\Omega_{\mathrm{H}}} I_{\mathrm{app}} \phi \mathrm{d} \boldsymbol{x}, \\
\int_{\Omega_{\mathrm{H}}}\left(\boldsymbol{\sigma}_{\mathrm{i}}+\boldsymbol{\sigma}_{\mathrm{e}}\right) \boldsymbol{\nabla} u_{\mathrm{e}} \cdot \boldsymbol{\nabla} \psi \mathrm{d} \boldsymbol{x}+\int_{\Omega_{\mathrm{H}}} \boldsymbol{\sigma}_{\mathrm{i}} \boldsymbol{\nabla} V_{\mathrm{m}} \cdot \boldsymbol{\nabla} \psi \mathrm{d} \boldsymbol{x}=0
\end{gathered}
$$

for all $(\xi, \phi, \psi) \in L^{2}\left(\Omega_{\mathrm{H}}\right) \times H^{1}\left(\Omega_{\mathrm{H}}\right) \times\left(H^{1}\left(\Omega_{\mathrm{H}}\right) \cap L_{0}^{2}\left(\Omega_{\mathrm{H}}\right)\right)$.

\subsection{Time semi-discrete formulations: decoupled time-mar- ching schemes}

Let $N \in \mathbb{N}^{*}$ be a given integer and consider a uniform partition $\left\{\left[t_{n}, t_{n+1}\right]\right\}_{0 \leq n \leq N-1}$, with $t_{n} \stackrel{\text { def }}{=} n \tau$, of the time interval of interest $(0, T)$, with time-step $\tau \stackrel{\text { def }}{=} T / N$. For a given time dependent function $X$, the quantity $X^{n}$ denotes an approximation of $X\left(t_{n}\right)$ and $D_{\tau} X^{n} \stackrel{\text { def }}{=}\left(X^{n}-X^{n-1}\right) / \tau$ the first order backward difference. Moreover, we set $I_{\text {app }}^{n} \stackrel{\text { def }}{=} I_{\text {app }}\left(t_{n}, \cdot\right)$.

We propose to time semi-discretize $(3.19$ by combining a first order semiimplicit treatment of the ionic current evaluation with an explicit (Gauss-Seidel or Jacobi like) treatment of the coupling between $V_{\mathrm{m}}$ and $u_{\mathrm{e}}$. The resulting schemes can be cast into a common frame as follows: For $0 \leq n \leq N-1$, we solve

1. Ionic state: find $w^{n+1} \in L^{\infty}\left(\Omega_{\mathrm{H}}\right)$ such that

$$
\int_{\Omega_{\mathrm{H}}}\left(D_{\tau} w^{n+1}+g\left(V_{\mathrm{m}}^{n}, w^{n+1}\right)\right) \xi \mathrm{d} \boldsymbol{x}=0
$$

for all $\xi \in L^{2}\left(\Omega_{\mathrm{H}}\right)$.

2. Transmembrane potential: find $V_{\mathrm{m}}^{n+1} \in H^{1}\left(\Omega_{\mathrm{H}}\right)$ such that

$$
\begin{array}{r}
\chi_{\mathrm{m}} \int_{\Omega_{\mathrm{H}}} D_{\tau} V_{\mathrm{m}}^{n+1} \phi \mathrm{d} \boldsymbol{x}+\int_{\Omega_{\mathrm{H}}} \boldsymbol{\sigma}_{\mathrm{i}} \boldsymbol{\nabla} V_{\mathrm{m}}^{n+1} \cdot \boldsymbol{\nabla} \phi \mathrm{d} \boldsymbol{x}+\int_{\Omega_{\mathrm{H}}} \boldsymbol{\sigma}_{\mathrm{i}} \boldsymbol{\nabla} u_{\mathrm{e}}^{\star} \cdot \boldsymbol{\nabla} \phi \mathrm{d} \boldsymbol{x} \\
=\int_{\Omega_{\mathrm{H}}}\left(I_{\mathrm{app}}^{n+1}-I_{\mathrm{ion}}\left(V_{\mathrm{m}}^{n}, w^{n+1}\right)\right) \phi \mathrm{d} \boldsymbol{x}
\end{array}
$$

for all $\phi \in H^{1}\left(\Omega_{\mathrm{H}}\right)$. 
3. Extracellular potential: find $u_{\mathrm{e}}^{n+1} \in H^{1}\left(\Omega_{\mathrm{H}}\right) \cap L_{0}^{2}\left(\Omega_{\mathrm{H}}\right)$,

$$
\int_{\Omega_{\mathrm{H}}}\left(\boldsymbol{\sigma}_{\mathrm{i}}+\boldsymbol{\sigma}_{\mathrm{e}}\right) \boldsymbol{\nabla} u_{\mathrm{e}}^{n+1} \cdot \boldsymbol{\nabla} \psi \mathrm{d} \boldsymbol{x}+\int_{\Omega_{\mathrm{H}}} \boldsymbol{\sigma}_{\mathrm{i}} \boldsymbol{\nabla} V_{\mathrm{m}}^{\star} \cdot \boldsymbol{\nabla} \psi \mathrm{d} \boldsymbol{x}=0
$$

for all $\psi \in H^{1}\left(\Omega_{\mathrm{H}}\right) \cap L_{0}^{2}\left(\Omega_{\mathrm{H}}\right)$.

Note that the non-linear system (3.20) and the ionic current evaluation $I_{\text {ion }}\left(V_{\mathrm{m}}^{n}, w^{n+1}\right)$ in (3.21) are decoupled from (3.21)-(3.22). This semi-implicit treatment is quite popular in the literature (see e.g. [45, 31, 14, 2, 5, 19]). For $\left(u_{\mathrm{e}}^{\star}, V_{\mathrm{m}}^{\star}\right)=\left(u_{\mathrm{e}}^{n+1}, V_{\mathrm{m}}^{n+1}\right)$, the unknown potentials $V_{\mathrm{m}}^{n+1}$ and $u_{\mathrm{e}}^{n+1}$ are implicit coupled and, therefore, equations (3.21) and (3.22) have to be solved simultaneously (see e.g. [27, 46, 19, 19, 4). The energy based stability analysis of this semi-implicit scheme, using first and second order time discretizations, has been recently reported in [19].

On the contrary, for $\left(u_{\mathrm{e}}^{\star}, V_{\mathrm{m}}^{\star}\right)=\left(u_{\mathrm{e}}^{n}, V_{\mathrm{m}}^{n+1}\right)$ or $\left(u_{\mathrm{e}}^{\star}, V_{\mathrm{m}}^{\star}\right)=\left(u_{\mathrm{e}}^{n}, V_{\mathrm{m}}^{n}\right)$, the electro-diffusive coupling becomes explicit and therefore (3.21) and $(3.22)$ can be solved separately: either sequentially (Gauss-Seidel) or in parallel (Jacobi). Similar Gauss-Seidel like splittings have been proposed and successfully applied in [45, 31, 2, 50. The theoretical stability of the schemes is, however, not established therein. To the best of our knowledge, the Jacobi like splitting has not yet been considered in the literature.

The energy based stability analysis of these time splitting schemes is performed in the next subsection.

Remark 3.1 The Gauss-Seidel and Jacobi like electro-diffusive splittings allow a decoupled computation of $V_{\mathrm{m}}$ and $u_{\mathrm{e}}$ without the need to resort to monodomain approximations (see Remark 2.1).

\subsection{Stability analysis}

For the stability analysis below, we shall make use of the following simplifying assumption (see [19, Section 3.2.2] and Remark 3.1 therein) on the structure of the ionic functions $g$ and $I_{\text {ion }}$ :

$$
\begin{gathered}
I_{\text {ion }}\left(V_{\mathrm{m}}, w\right) \leq C_{I}\left(\left|V_{\mathrm{m}}\right|+|w|\right), \\
g\left(V_{\mathrm{m}}, w\right) \leq C_{g}\left(\left|V_{\mathrm{m}}\right|+|w|\right)
\end{gathered}
$$

for all $V_{\mathrm{m}}, w$, and we set $\alpha \stackrel{\text { def }}{=} 1+3 C_{I}+C_{g}$ and $\beta \stackrel{\text { def }}{=} C_{I}+3 C_{g}$.

The next theorem states the energy based stability of the time-marching schemes $3.20-(3.22)$, in terms of $u_{\mathrm{e}}^{\star}$ and $V_{\mathrm{m}}^{\star}$.

Theorem 3.2 Assume that (3.23) holds and that the conductivity tensors $\boldsymbol{\sigma}_{\mathrm{i}}, \boldsymbol{\sigma}_{\mathrm{e}}$ are symmetric and positive-definite. Let $\left\{\left(w^{n}, V_{\mathrm{m}}^{n}, u_{\mathrm{e}}^{n}\right)\right\}_{n=0}^{N}$ be solution of (3.20)(3.22) and $C(T, \alpha, \beta) \stackrel{\text { def }}{=} \exp (T /(1-\tau \max \{\alpha, \beta\}))$. Then, under the condition

$$
\tau<\frac{1}{\max \{\alpha, \beta\}}
$$

there follows: 
- $\operatorname{For}\left(u_{\mathrm{e}}^{\star}, V_{\mathrm{m}}^{\star}\right)=\left(u_{\mathrm{e}}^{n+1}, V_{\mathrm{m}}^{n+1}\right)$ :

$$
\begin{aligned}
& \left\|w^{n}\right\|_{0, \Omega_{\mathrm{H}}}^{2}+\chi_{\mathrm{m}}\left\|V_{\mathrm{m}}^{n}\right\|_{0, \Omega_{\mathrm{H}}}^{2}+2 \sum_{m=0}^{n-1} \tau\left\|\boldsymbol{\sigma}_{\mathrm{e}}^{\frac{1}{2}} \nabla u_{\mathrm{e}}^{m+1}\right\|_{0, \Omega_{\mathrm{H}}}^{2}+2 \sum_{m=0}^{n-1} \tau\left\|\boldsymbol{\sigma}_{\mathrm{i}}^{\frac{1}{2}} \nabla\left(V_{\mathrm{m}}^{m+1}+u_{\mathrm{e}}^{m+1}\right)\right\|_{0, \Omega_{\mathrm{H}}}^{2} \\
& \leq C(T, \alpha, \beta)\left(\left\|w^{0}\right\|_{0, \Omega_{\mathrm{H}}}^{2}+\chi_{\mathrm{m}}\left\|V_{\mathrm{m}}^{0}\right\|_{0, \Omega_{\mathrm{H}}}^{2}+\sum_{m=0}^{n-1} \tau\left\|I_{\mathrm{app}}^{m+1}\right\|_{0, \Omega_{\mathrm{H}}}^{2}\right),
\end{aligned}
$$

with $1 \leq n \leq N$.

- $\operatorname{For}\left(u_{\mathrm{e}}^{\star}, V_{\mathrm{m}}^{\star}\right)=\left(u_{\mathrm{e}}^{n}, V_{\mathrm{m}}^{n+1}\right)$ :

$$
\begin{aligned}
& \left\|w^{n}\right\|_{0, \Omega_{\mathrm{H}}}^{2}+\chi_{\mathrm{m}}\left\|V_{\mathrm{m}}^{n}\right\|_{0, \Omega_{\mathrm{H}}}^{2}+\tau\left\|\boldsymbol{\sigma}_{\mathrm{i}}^{\frac{1}{2}} \nabla u_{\mathrm{e}}^{n}\right\|_{0, \Omega_{\mathrm{H}}}^{2} \\
& \quad+2 \sum_{m=0}^{n-1} \tau\left\|\boldsymbol{\sigma}_{\mathrm{e}}^{\frac{1}{2}} \nabla u_{\mathrm{e}}^{m+1}\right\|_{0, \Omega_{\mathrm{H}}}^{2}+\sum_{m=0}^{n-1} \tau\left\|\boldsymbol{\sigma}_{\mathrm{i}}^{\frac{1}{2}} \nabla\left(V_{\mathrm{m}}^{m+1}+u_{\mathrm{e}}^{m+1}\right)\right\|_{0, \Omega_{\mathrm{H}}}^{2} \\
& \leq C(T, \alpha, \beta)\left(\left\|w^{0}\right\|_{0, \Omega_{\mathrm{H}}}^{2}+\chi_{\mathrm{m}}\left\|V_{\mathrm{m}}^{0}\right\|_{0, \Omega_{\mathrm{H}}}^{2}+\tau\left\|\boldsymbol{\sigma}_{\mathrm{i}}^{\frac{1}{2}} \nabla u_{\mathrm{e}}^{0}\right\|_{0, \Omega_{\mathrm{H}}}^{2}+\sum_{m=0}^{n-1} \tau\left\|I_{\mathrm{app}}^{m+1}\right\|_{0, \Omega_{\mathrm{H}}}^{2}\right),
\end{aligned}
$$

with $1 \leq n \leq N$.

- $\operatorname{For}\left(u_{\mathrm{e}}^{\star}, V_{\mathrm{m}}^{\star}\right)=\left(u_{\mathrm{e}}^{n}, V_{\mathrm{m}}^{n}\right)$ :

$$
\begin{aligned}
& \left\|w^{n}\right\|_{0, \Omega_{\mathrm{H}}}^{2}+\chi_{\mathrm{m}}\left\|V_{\mathrm{m}}^{n}\right\|_{0, \Omega_{\mathrm{H}}}^{2}+\tau\left\|\boldsymbol{\sigma}_{\mathrm{i}}^{\frac{1}{2}} \nabla u_{\mathrm{e}}^{n}\right\|_{0, \Omega_{\mathrm{H}}}^{2}+\tau\left\|\boldsymbol{\sigma}_{\mathrm{i}}^{\frac{1}{2}} \nabla V_{\mathrm{m}}^{n}\right\|_{0, \Omega_{\mathrm{H}}}^{2} \\
& +2 \sum_{m=0}^{n-1} \tau\left\|\boldsymbol{\sigma}_{\mathrm{e}}^{\frac{1}{2}} \nabla u_{\mathrm{e}}^{m+1}\right\|_{0, \Omega_{\mathrm{H}}}^{2} \leq C(T, \alpha, \beta)\left(\left\|w^{0}\right\|_{0, \Omega_{\mathrm{H}}}^{2}+\chi_{\mathrm{m}}\left\|V_{\mathrm{m}}^{0}\right\|_{0, \Omega_{\mathrm{H}}}^{2}\right. \\
& \left.\quad+\tau\left\|\boldsymbol{\sigma}_{\mathrm{i}}^{\frac{1}{2}} \nabla V_{\mathrm{m}}^{0}\right\|_{0, \Omega_{\mathrm{H}}}^{2}+\tau\left\|\boldsymbol{\sigma}_{\mathrm{i}}^{\frac{1}{2}} \nabla u_{\mathrm{e}}^{0}\right\|_{0, \Omega_{\mathrm{H}}}^{2}+\sum_{m=0}^{n-1} \tau\left\|I_{\mathrm{app}}^{m+1}\right\|_{0, \Omega_{\mathrm{H}}}^{2}\right),
\end{aligned}
$$

with $1 \leq n \leq N$.

Proof. See appendix $\$$ A.1.

We conclude this section with a series of remarks.

Remark 3.3 Theorem 3.2 shows that electro-diffusive Gauss-Seidel and Jacobi splittings are energy stable under condition (3.24), as for the unsplit case $\left(u_{\mathrm{e}}^{\star}, V_{\mathrm{m}}^{\star}\right)=\left(u_{\mathrm{e}}^{n+1}, V_{\mathrm{m}}^{n+1}\right)$ (analyzed in [19]), but with slightly altered energy norms. As a result, stability is not compromised.

Remark 3.4 The proof of Theorem 3.2 (see appendix $\$ A .1$ ) does not depend on the time discretization considered in (3.20) and (3.21). Indeed, we do not make use of any numerical dissipation produced by the scheme, a part from that is directly provided by the splitting. Therefore, the backward Euler quotients, $D_{\tau} w^{n+1}$ and $D_{\tau} V_{\mathrm{m}}^{n+1}$, can be safely replaced by a second order backward difference formula, and perform one correction (see e.g. [43, 41]) to recover overall second order accuracy. 
Remark 3.5 The above stability result can be adapted, with minor modifications, to the case $\left(u_{\mathrm{e}}^{\star}, V_{\mathrm{m}}^{\star}\right)=\left(u_{\mathrm{e}}^{n+1}, V_{\mathrm{m}}^{n}\right)$. The full Jacobi splitting, obtained after replacing $I_{\mathrm{ion}}\left(V_{\mathrm{m}}^{n}, w^{n+1}\right)$ by $I_{\mathrm{ion}}\left(V_{\mathrm{m}}^{n}, w^{n}\right)$ in (3.21) could also be considered.

Remark 3.6 Theorem 3.2 holds also for the fully discrete counterpart of (3.20)(3.22) obtained by discretizing in space using finite elements (see subsection \$.1.

\section{Decoupled time-marching for ECG numerical simulation}

In this section, we introduce fully discrete schemes for the heart-torso system (2.8)-2.16), allowing a decoupled computation of the transmembrane, extracellular and torso potentials. The main idea consists in combining the bidomain splittings of the previous section, with a specific explicit Robin-Robin treatment of the heart-torso coupling conditions $(2.14)-(2.15)$.

\subsection{Preliminaries}

Problem 2.8 -2.16 can be cast into weak form as follows (see e.g. [6]): for $t>0$, find $w(\cdot, t) \in L^{\infty}\left(\Omega_{\mathrm{H}}\right), V_{\mathrm{m}}(\cdot, t) \in H^{1}\left(\Omega_{\mathrm{H}}\right), u_{\mathrm{e}}(\cdot, t) \in H^{1}\left(\Omega_{\mathrm{H}}\right) \cap L_{0}^{2}\left(\Omega_{\mathrm{H}}\right)$ and $u_{\mathrm{T}}(\cdot, t) \in H^{1}\left(\Omega_{\mathrm{T}}\right)$ with $u_{\mathrm{e}}(\cdot, t)=u_{\mathrm{T}}(\cdot, t)$ on $\Sigma$, such that

$$
\begin{array}{r}
\int_{\Omega_{\mathrm{H}}}\left(\partial_{t} w+g\left(V_{\mathrm{m}}, w\right)\right) \xi \mathrm{d} \boldsymbol{x}=0, \\
\chi_{\mathrm{m}} \int_{\Omega_{\mathrm{H}}}\left(\partial_{t} V_{\mathrm{m}}+I_{\mathrm{ion}}\left(V_{\mathrm{m}}, w\right)\right) \phi \mathrm{d} \boldsymbol{x}+\int_{\Omega_{\mathrm{H}}} \boldsymbol{\sigma}_{\mathrm{i}} \boldsymbol{\nabla}\left(V_{\mathrm{m}}+u_{\mathrm{e}}\right) \cdot \boldsymbol{\nabla} \phi \mathrm{d} \boldsymbol{x}=\int_{\Omega_{\mathrm{H}}} I_{\mathrm{app}} \phi \mathrm{d} \boldsymbol{x}, \\
\int_{\Omega_{\mathrm{H}}}\left(\boldsymbol{\sigma}_{\mathrm{i}}+\boldsymbol{\sigma}_{\mathrm{e}}\right) \boldsymbol{\nabla} u_{\mathrm{e}} \cdot \nabla \psi \mathrm{d} \boldsymbol{x}+\int_{\Omega_{\mathrm{H}}} \boldsymbol{\sigma}_{\mathrm{i}} \nabla V_{\mathrm{m}} \cdot \nabla \psi \mathrm{d} \boldsymbol{x}+\int_{\Omega_{\mathrm{T}}} \boldsymbol{\sigma}_{\mathrm{T}} \boldsymbol{\nabla} u_{\mathrm{T}} \cdot \boldsymbol{\nabla} \zeta \mathrm{d} \boldsymbol{x}=0
\end{array}
$$

for all $(\xi, \phi, \psi, \zeta) \in L^{2}\left(\Omega_{\mathrm{H}}\right) \times H^{1}\left(\Omega_{\mathrm{H}}\right) \times\left(H^{1}\left(\Omega_{\mathrm{H}}\right) \cap L_{0}^{2}\left(\Omega_{\mathrm{H}}\right)\right) \times H^{1}\left(\Omega_{\mathrm{T}}\right)$ with $\psi=\zeta$ on $\Sigma$.

Assume that $\Omega_{\mathrm{H}}$ and $\Omega_{\mathrm{T}}$ are polygonal domains and let $\left\{\mathcal{T}_{\mathrm{H}, h}\right\}_{0<h \leq 1}\left(\left\{\mathcal{T}_{\mathrm{T}, h}\right\}_{0<h \leq 1}\right)$ be a family of triangulations of $\Omega_{\mathrm{H}}\left(\operatorname{resp} . \Omega_{\mathrm{T}}\right)$ satisfying the usual requirements of finite element approximations (see e.g. [18]). The subscript $h \in(0,1]$ refer to the level of refinement of the triangulations. For the sake of simplicity and without loss of generality, we assume that both families of triangulations are quasi-uniform and that they match at the heart-torso interface $\Sigma$. We define $X_{\mathrm{H}, h}$ (resp. $\left.X_{\mathrm{T}, h}\right)$ as an internal continuous Lagrange finite element approximation of $H^{1}\left(\Omega_{\mathrm{H}}\right)$ (resp. $H^{1}\left(\Omega_{\mathrm{T}}\right)$ ). We also introduce the standard finite element (heart-to-torso) lifting operator $\mathcal{L}_{h}: X_{\mathrm{H}, h} \rightarrow X_{\mathrm{T}, h} \cap H_{\Gamma_{\text {ext }}}^{1}\left(\Omega_{\mathrm{T}}\right)$, such that $\mathcal{L}_{h} \psi=\psi$ on $\Sigma$ and $\mathcal{L}_{h} \psi=0$ on $\Gamma_{\text {ext }}$, for all $\psi \in X_{\mathrm{H}, h}$. Note that we have the direct sum decomposition

$$
\left\{(\psi, \zeta) \in X_{\mathrm{H}, h} \times X_{\mathrm{T}, h}: \psi_{\mid \Sigma}=\zeta_{\mid \Sigma}\right\}=\left\{\left(\psi, \mathcal{L}_{h} \psi\right): \psi \in X_{\mathrm{H}, h}\right\} \oplus\left\{(0, \zeta): \zeta \in X_{\mathrm{T}, h} \cap H_{\Sigma}^{1}\left(\Omega_{\mathrm{T}}\right)\right\} .
$$


In the stability analysis below, we shall make use of the following standard discrete trace-inverse inequality (see e.g. [47]):

$$
\|v\|_{0, \partial K}^{2} \leq \frac{C_{\mathrm{ti}}}{h}\|v\|_{0, K}^{2} \quad \forall v \in X_{\mathrm{T}, h}
$$

for all $K \in \mathcal{T}_{\mathrm{T}, h}$, and with $C_{\mathrm{ti}}>0$ a constant independent of the discretization parameter $h$ (but that might depend on the polynomial order).

By combining the semi-implicit time-marching schemes of section 3 with a finite element discretization in space, we can derive from 4.28 the following fully discrete heart-torso scheme: for $0 \leq n \leq N-1$, find $w^{n+1} \in X_{\mathrm{H}, h}$, $V_{\mathrm{m}}^{n+1} \in X_{\mathrm{H}, h}, u_{\mathrm{e}}^{n+1} \in X_{\mathrm{H}, h} \cap L_{0}^{2}\left(\Omega_{\mathrm{H}}\right)$ and $u_{\mathrm{T}}^{n+1} \in X_{\mathrm{T}, h}$ with $u_{\mathrm{T}}^{n+1}=u_{\mathrm{e}}^{n+1}$ on $\Sigma$, such that

$$
\begin{gathered}
\int_{\Omega_{\mathrm{H}}}\left(D_{\tau} w^{n+1}+g\left(V_{\mathrm{m}}^{n}, w^{n+1}\right)\right) \xi \mathrm{d} \boldsymbol{x}=0, \\
\chi_{\mathrm{m}} \int_{\Omega_{\mathrm{H}}} D_{\tau} V_{\mathrm{m}}^{n+1} \phi \mathrm{d} \boldsymbol{x}+\int_{\Omega_{\mathrm{H}}} \boldsymbol{\sigma}_{\mathrm{i}} \boldsymbol{\nabla}\left(V_{\mathrm{m}}^{n+1}+u_{\mathrm{e}}^{\star}\right) \cdot \nabla \phi \mathrm{d} \boldsymbol{x}=\int_{\Omega_{\mathrm{H}}}\left(I_{\mathrm{app}}^{n+1}-I_{\mathrm{ion}}\left(V_{\mathrm{m}}^{n}, w^{n+1}\right)\right) \phi \mathrm{d} \boldsymbol{x}, \\
\int_{\Omega_{\mathrm{H}}}\left(\boldsymbol{\sigma}_{\mathrm{i}}+\boldsymbol{\sigma}_{\mathrm{e}}\right) \boldsymbol{\nabla} u_{\mathrm{e}}^{n+1} \cdot \nabla \psi \mathrm{d} \boldsymbol{x}+\int_{\Omega_{\mathrm{H}}} \boldsymbol{\sigma}_{\mathrm{i}} \nabla V_{\mathrm{m}}^{\star} \cdot \nabla \psi \mathrm{d} \boldsymbol{x}+\int_{\Omega_{\mathrm{T}}} \boldsymbol{\sigma}_{\mathrm{T}} \boldsymbol{\nabla} u_{\mathrm{T}}^{n+1} \cdot \boldsymbol{\nabla} \zeta \mathrm{d} \boldsymbol{x}=0
\end{gathered}
$$

for all $(\xi, \phi, \psi, \zeta) \in X_{\mathrm{H}, h} \times X_{\mathrm{H}, h} \times\left(X_{\mathrm{H}, h} \cap L_{0}^{2}\left(\Omega_{\mathrm{H}}\right)\right) \times X_{\mathrm{T}, h}$ with $\zeta=\psi$ on $\Sigma$. Equivalently, using (4.29), the heart-torso subproblem 4.33 can be split into two coupled, torso (Dirichlet) and heart (Neumann), subproblems as follows:

- Find $u_{\mathrm{T}}^{n+1} \in X_{\mathrm{T}, h}$, with $u_{\mathrm{T}}^{n+1}=u_{\mathrm{e}}^{n+1}$ on $\Sigma$, such that

$$
\int_{\Omega_{\mathrm{T}}} \boldsymbol{\sigma}_{\mathrm{T}} \boldsymbol{\nabla} u_{\mathrm{T}}^{n+1} \cdot \nabla \zeta \mathrm{d} \boldsymbol{x}=0
$$

for all $\zeta \in X_{\mathrm{T}, h}$, with $\zeta=0$ on $\Sigma$.

- Find $u_{\mathrm{e}}^{n+1} \in X_{\mathrm{H}, h} \cap L_{0}^{2}\left(\Omega_{\mathrm{H}}\right)$ such that

$$
\int_{\Omega_{\mathrm{H}}}\left(\boldsymbol{\sigma}_{\mathrm{i}}+\boldsymbol{\sigma}_{\mathrm{e}}\right) \boldsymbol{\nabla} u_{\mathrm{e}}^{n+1} \cdot \boldsymbol{\nabla} \psi \mathrm{d} \boldsymbol{x}+\int_{\Omega_{\mathrm{H}}} \boldsymbol{\sigma}_{\mathrm{i}} \boldsymbol{\nabla} V_{\mathrm{m}}^{\star} \cdot \boldsymbol{\nabla} \psi \mathrm{d} \boldsymbol{x}=-\int_{\Omega_{\mathrm{T}}} \boldsymbol{\sigma}_{\mathrm{T}} \boldsymbol{\nabla} u_{\mathrm{T}}^{n+1} \cdot \boldsymbol{\nabla} \mathcal{L}_{h} \psi \mathrm{d} \boldsymbol{x}
$$

for all $\psi \in X_{\mathrm{H}, h} \cap L_{0}^{2}\left(\Omega_{\mathrm{H}}\right)$.

Remark 4.1 The residual term in the right hand side of 4.35 amounts to enforce the Neumann condition 2.15 in a variational consistent fashion.

Despite the Gauss-Seidel and Jacobi splittings allow a decoupled solution of (4.31) and 4.32), the heart and torso potentials $u_{\mathrm{e}}^{n+1}$ and $u_{\mathrm{T}}^{n+1}$ are still implicitly coupled. Therefore, problems (4.34) and 4.35 must be solved simultaneously: either monolithically, after assembling of (4.33) (see e.g. [45, 31, 46, 50]) or, in a partitioned fashion, by sub-iterating between them (see e.g. [9, 4]). Note that, since the (quasi-static) time discretization 4.34 and 4.35 do not 
generate numerical dissipation in time, the naive Dirichlet-Neumann explicit coupling, obtained by enforcing

$$
u_{\mathrm{T}}^{n+1}=u_{\mathrm{e}}^{n} \quad \text { on } \quad \Sigma,
$$

in the torso subproblem 4.34), might lead to numerical instability.

In the next subsection, we introduce an alternative heart-torso splitting, based on Robin-Robin transmission conditions, which remains stable under a hyperbolic-CFL like condition.

\subsection{Fully discrete formulation: decoupled time-marching schemes}

We propose to combine the decoupling techniques introduced in section $\$ 3$ with the following explicit Robin-Robin splitting, derived from [1] (see also [10]), of the heart-torso coupling:

$$
\begin{gathered}
\boldsymbol{\sigma}_{\mathrm{e}} \boldsymbol{\nabla} u_{\mathrm{e}}^{n+1} \cdot \boldsymbol{n}+\frac{\gamma \sigma_{\mathrm{T}}^{\mathrm{t}}}{h} u_{\mathrm{e}}^{n+1}=-\boldsymbol{\sigma}_{\mathrm{T}} \boldsymbol{\nabla} u_{\mathrm{T}}^{n} \cdot \boldsymbol{n}_{\mathrm{T}}+\frac{\gamma \sigma_{\mathrm{T}}^{\mathrm{t}}}{h} u_{\mathrm{T}}^{n}, \quad \text { on } \quad \Sigma, \\
\boldsymbol{\sigma}_{\mathrm{T}} \boldsymbol{\nabla} u_{\mathrm{T}}^{n+1} \cdot \boldsymbol{n}_{\mathrm{T}}+\frac{\gamma \sigma_{\mathrm{T}}^{\mathrm{t}}}{h} u_{\mathrm{T}}^{n+1}=\boldsymbol{\sigma}_{\mathrm{T}} \boldsymbol{\nabla} u_{\mathrm{T}}^{n} \cdot \boldsymbol{n}_{\mathrm{T}}+\frac{\gamma \sigma_{\mathrm{T}}^{\mathrm{t}}}{h} u_{\mathrm{e}}^{n+1}, \quad \text { on } \quad \Sigma,
\end{gathered}
$$

where $\gamma>0$ is a free Robin parameter, to be specified later on, and $\sigma_{\mathrm{T}}^{\mathrm{t}}$ is such that $\boldsymbol{\sigma}_{\mathrm{T} \mid \Sigma}=\sigma_{\mathrm{T}}^{\mathrm{t}} \boldsymbol{I}$.

Remark 4.2 We have assumed, without loss of generality, that the torso conductivity tensor is isotropic on the boundary, $\boldsymbol{\sigma}_{\mathrm{T} \mid \Sigma}=\sigma_{\mathrm{T}}^{\mathrm{t}} \boldsymbol{I}$, and that $\sigma_{\mathrm{T}}^{\mathrm{t}}$ is constant.

Thus, the resulting schemes read as follows: for $0 \leq n \leq N-1$, we solve:

1. Ionic state: find $w^{n+1} \in X_{h}$ such that

$$
\int_{\Omega_{\mathrm{H}}}\left(D_{\tau} w^{n+1}+g\left(V_{\mathrm{m}}^{n}, w^{n+1}\right)\right) \xi \mathrm{d} \boldsymbol{x}=0
$$

for all $\xi \in X_{h}$.

2. Transmembrane potential: find $V_{\mathrm{m}}^{n+1} \in X_{h}$ such that

$$
\begin{array}{r}
\chi_{\mathrm{m}} \int_{\Omega_{\mathrm{H}}} D_{\tau} V_{\mathrm{m}}^{n+1} \phi \mathrm{d} \boldsymbol{x}+\int_{\Omega_{\mathrm{H}}} \boldsymbol{\sigma}_{\mathrm{i}} \boldsymbol{\nabla} V_{\mathrm{m}}^{n+1} \cdot \boldsymbol{\nabla} \phi \mathrm{d} \boldsymbol{x}+\int_{\Omega_{\mathrm{H}}} \boldsymbol{\sigma}_{\mathrm{i}} \boldsymbol{\nabla} u_{\mathrm{e}}^{\star} \cdot \boldsymbol{\nabla} \phi \mathrm{d} \boldsymbol{x} \\
=\int_{\Omega_{\mathrm{H}}}\left(I_{\mathrm{app}}^{n+1}-I_{\mathrm{ion}}\left(V_{\mathrm{m}}^{n}, w^{n+1}\right)\right) \phi \mathrm{d} \boldsymbol{x}
\end{array}
$$

for all $\phi \in X_{h}$.

3. Extracellular potential: find $u_{\mathrm{e}}^{n+1} \in X_{h}$ such that

$$
\begin{array}{r}
\int_{\Omega_{\mathrm{H}}}\left(\boldsymbol{\sigma}_{\mathrm{i}}+\boldsymbol{\sigma}_{\mathrm{e}}\right) \boldsymbol{\nabla} u_{\mathrm{e}}^{n+1} \cdot \boldsymbol{\nabla} \psi \mathrm{d} \boldsymbol{x}+\int_{\Omega_{\mathrm{H}}} \boldsymbol{\sigma}_{\mathrm{i}} \boldsymbol{\nabla} V_{\mathrm{m}}^{\star} \cdot \boldsymbol{\nabla} \psi \mathrm{d} \boldsymbol{x}+\frac{\gamma \sigma_{\mathrm{T}}^{\mathrm{t}}}{h} \int_{\Sigma} u_{\mathrm{e}}^{n+1} \psi \mathrm{d} \boldsymbol{s} \\
=-\int_{\Sigma} \boldsymbol{\sigma}_{\mathrm{T}} \boldsymbol{\nabla} u_{\mathrm{T}}^{n} \cdot \boldsymbol{n}_{\mathrm{T}} \psi \mathrm{d} \boldsymbol{s}+\frac{\gamma \sigma_{\mathrm{T}}^{\mathrm{t}}}{h} \int_{\Sigma} u_{\mathrm{T}}^{n} \psi \mathrm{d} \boldsymbol{s}
\end{array}
$$

for all $\psi \in X_{h}$. 
4. Torso potential: find $u_{\mathrm{T}}^{n+1} \in X_{h}$

$$
\int_{\Omega_{\mathrm{T}}} \boldsymbol{\sigma}_{\mathrm{T}} \boldsymbol{\nabla} u_{\mathrm{T}}^{n+1} \cdot \boldsymbol{\nabla} \zeta \mathrm{d} \boldsymbol{x}+\frac{\gamma \sigma_{\mathrm{T}}^{\mathrm{t}}}{h} \int_{\Sigma} u_{\mathrm{T}}^{n+1} \zeta \mathrm{d} \boldsymbol{s}=\int_{\Sigma} \boldsymbol{\sigma}_{\mathrm{T}} \boldsymbol{\nabla} u_{\mathrm{T}}^{n} \cdot \boldsymbol{n}_{\mathrm{T}} \zeta \mathrm{d} \boldsymbol{s}+\frac{\gamma \sigma_{\mathrm{T}}^{\mathrm{t}}}{h} \int_{\Sigma} u_{\mathrm{e}}^{n+1} \zeta \mathrm{d} \boldsymbol{s}
$$

for all $\zeta \in X_{h}$.

Contrarily to 4.31- 4.33 , the cardiac subproblem 4.37)- 4.39) can be solved independently of the torso subproblem (4.40). In particular, the choices $\left(u_{\mathrm{e}}^{\star}, V_{\mathrm{m}}^{\star}\right)=\left(u_{\mathrm{e}}^{n}, V_{\mathrm{m}}^{n+1}\right)$ or $\left(u_{\mathrm{e}}^{\star}, V_{\mathrm{m}}^{\star}\right)=\left(u_{\mathrm{e}}^{n}, V_{\mathrm{m}}^{n}\right)$ lead to a fully decoupled computation of $w^{n+1}, V_{\mathrm{m}}^{n+1}, u_{\mathrm{e}}^{n+1}$ and $u_{\mathrm{T}}^{n+1}$. In other words, the four subproblems 4.37)-4.40 are decoupled and can be solved sequentially.

The energy based numerical stability of these schemes is addressed in the next subsection.

Remark 4.3 The choices $\left(u_{\mathrm{e}}^{\star}, V_{\mathrm{m}}^{\star}\right)=\left(u_{\mathrm{e}}^{n}, V_{\mathrm{m}}^{n+1}\right)$ or $\left(u_{\mathrm{e}}^{\star}, V_{\mathrm{m}}^{\star}\right)=\left(u_{\mathrm{e}}^{n}, V_{\mathrm{m}}^{n}\right)$ in 4.37)-4.40 allow a fully decoupled computation of $w^{n+1}, V_{\mathrm{m}}^{n+1}, u_{\mathrm{e}}^{n+1}$ and $u_{\mathrm{T}}^{n+1}$ without the need to resort to monodomain and uncoupling approximations (see Remark 2.3).

\subsection{Stability analysis}

We address here the energy based stability of the heart-torso coupling schemes 4.37)-4.40 . Hence, in order to alleviate the exposition, we denote by $E_{\mathrm{H}}^{0}\left(u_{\mathrm{e}}^{\star}, V_{\mathrm{m}}^{\star}\right)$ (resp. $\left.E_{\mathrm{H}}^{n}\left(u_{\mathrm{e}}^{\star}, V_{\mathrm{m}}^{\star}\right)\right)$ the discrete bidomain energy at steps 0 (resp. $n$ ), arising in the stability estimates provided by Theorem 3.2. For instance, in the case $\left(u_{\mathrm{e}}^{\star}, V_{\mathrm{m}}^{\star}\right)=\left(u_{\mathrm{e}}^{n}, V_{\mathrm{m}}^{n}\right)$, we have

$$
\begin{gathered}
E_{\mathrm{H}}^{0}\left(u_{\mathrm{e}}^{\star}, V_{\mathrm{m}}^{\star}\right) \stackrel{\text { def }}{=}\left\|w^{0}\right\|_{0, \Omega_{\mathrm{H}}}^{2}+\chi_{\mathrm{m}}\left\|V_{\mathrm{m}}^{0}\right\|_{0, \Omega_{\mathrm{H}}}^{2}+\tau\left\|\boldsymbol{\sigma}_{\mathrm{i}}^{\frac{1}{2}} \nabla V_{\mathrm{m}}^{0}\right\|_{0, \Omega_{\mathrm{H}}}^{2}+\tau\left\|\boldsymbol{\sigma}_{\mathrm{i}}^{\frac{1}{2}} \nabla u_{\mathrm{e}}^{0}\right\|_{0, \Omega_{\mathrm{H}}}^{2}, \\
E_{\mathrm{H}}^{n}\left(u_{\mathrm{e}}^{\star}, V_{\mathrm{m}}^{\star}\right) \stackrel{\text { def }}{=}\left\|w^{n}\right\|_{0, \Omega_{\mathrm{H}}}^{2}+\chi_{\mathrm{m}}\left\|V_{\mathrm{m}}^{n}\right\|_{0, \Omega_{\mathrm{H}}}^{2}+\tau\left\|\boldsymbol{\sigma}_{\mathrm{i}}^{\frac{1}{2}} \nabla u_{\mathrm{e}}^{n}\right\|_{0, \Omega_{\mathrm{H}}}^{2}+\tau\left\|\boldsymbol{\sigma}_{\mathrm{i}}^{\frac{1}{2}} \nabla V_{\mathrm{m}}^{n}\right\|_{0, \Omega_{\mathrm{H}}}^{2} \\
+2 \sum_{m=0}^{n-1} \tau\left\|\boldsymbol{\sigma}_{\mathrm{e}}^{\frac{1}{2}} \nabla u_{\mathrm{e}}^{m+1}\right\|_{0, \Omega_{\mathrm{H}}}^{2},
\end{gathered}
$$

and similarly for the rest.

The next theorem states the main result of this section.

Theorem 4.4 Assume that the hypothesis of Theorem 3.2 hold and that the torso conductivity tensor $\boldsymbol{\sigma}_{\mathrm{T}}$ is symmetric and positive-definite. Let $\left\{\left(w^{n}, V_{\mathrm{m}}^{n}, u_{\mathrm{e}}^{n}, u_{\mathrm{T}}^{n}\right)\right\}_{n=0}^{N}$ be solution of 4.37)-4.40. Then for

$$
\gamma>2 C_{\mathrm{ti}}
$$

the following estimate holds

$$
\begin{aligned}
& E_{\mathrm{H}}^{n}\left(u_{\mathrm{e}}^{\star}, V_{\mathrm{m}}^{\star}\right)+\tau \frac{\gamma \sigma_{\mathrm{T}}^{\mathrm{t}}}{h}\left\|u_{\mathrm{T}}^{n}\right\|_{0, \Sigma}^{2}+\sum_{m=0}^{n-1} \tau\left\|\boldsymbol{\sigma}_{\mathrm{T}}^{\frac{1}{2}} \nabla u_{\mathrm{T}}^{m+1}\right\|_{0, \Omega_{\mathrm{T}}}^{2}+\sum_{m=0}^{n-1} \tau \frac{\gamma \sigma_{\mathrm{T}}^{\mathrm{t}}}{2 h}\left\|u_{\mathrm{T}}^{m+1}-u_{\mathrm{e}}^{m+1}\right\|_{0, \Sigma}^{2} \\
\leq & C(T, \alpha, \beta)\left(E_{\mathrm{H}}^{0}\left(u_{\mathrm{e}}^{\star}, V_{\mathrm{m}}^{\star}\right)+\tau \frac{\gamma \sigma_{\mathrm{T}}^{\mathrm{t}}}{h}\left\|u_{\mathrm{T}}^{0}\right\|_{0, \Sigma}^{2}+\tau\left\|\boldsymbol{\sigma}_{\mathrm{T}}^{\frac{1}{2}} \nabla u_{\mathrm{T}}^{0}\right\|_{0, \Omega_{\mathrm{T}}}^{2}+\sum_{m=0}^{n-1} \tau\left\|I_{\mathrm{app}}^{m+1}\right\|_{0, \Omega_{\mathrm{H}}}^{2}\right),
\end{aligned}
$$


with $1 \leq n \leq N$. In particular, 4.42 ensures the energy based stability of the explicit heart-torso coupling 4.37)-(4.40) under the condition $\tau=O(h)$.

Proof. See appendix $\$$ A.2.

Remark 4.5 The above proof does not make use of any numerical dissipation apart from that directly provided by the explicit Robin-Robin splitting (4.36). Note that this is particularly well adapted to the heart-torso coupling (2.8)(2.16), since the quasi-static elliptic equations 2.9 and $(2.10)$ do not generate numerical dissipation in time.

Remark 4.6 The flux terms in 4.39 and 4.40 can be evaluated face-wise, i.e. as broken integrals, or using a discrete variational expression, as in (4.35). Hence, $\int_{\Sigma} \boldsymbol{\sigma}_{\mathrm{T}} \boldsymbol{\nabla} u_{\mathrm{T}}^{n} \cdot \boldsymbol{n}_{\mathrm{T}} \psi$ and $\int_{\Sigma} \boldsymbol{\sigma}_{\mathrm{T}} \boldsymbol{\nabla} u_{\mathrm{T}}^{n} \cdot \boldsymbol{n}_{\mathrm{T}} \zeta$, can be safely replaced by $\int_{\Omega_{\mathrm{T}}} \boldsymbol{\sigma}_{\mathrm{T}} \boldsymbol{\nabla} u_{\mathrm{T}}^{n}$. $\boldsymbol{\nabla} \mathcal{L}_{h} \psi$ and $\int_{\Omega_{\mathrm{T}}} \boldsymbol{\sigma}_{\mathrm{T}} \boldsymbol{\nabla} u_{\mathrm{T}}^{n} \cdot \boldsymbol{\nabla} \mathcal{L}_{h} \zeta$, respectively.

\section{$5 \quad$ Numerical results}

In this section we illustrate, via numerical experiments, the stability and accuracy of the time decoupling schemes analyzed in the previous sections. Subsection 5.1 reports on numerical investigations with an academic mono-dimensional test problem. Numerical results based on anatomical heart and torso geometries are presented in subsection $\$ 5.2$

\subsection{Mono-dimensional study}

In this subsection we illustrate numerically the convergence behavior of the decoupling schemes considered above. We limit the study to the mono-dimensional case, which allows to consider highly accurate reference solutions at a moderate computational cost. Subsection $\$ 5.1 .2$ is devoted to electro-diffusive splittings analyzed in section $\$ 3$, whereas the convergence of the heart-torso decoupling schemes, introduced in section $\$ 4$ is investigated in subsection $\$ 5.1 .3$

\subsubsection{Simulation data}

We consider a variant of the mono-dimensional test case introduced in [19, Section 4.1], all parameters and quantities are dimensionless. In (2.1)-(2.6) and 2.8)-2.16, we take $\Omega_{\mathrm{H}}=[0, L], \Omega_{\mathrm{T}}=[L, 2 L], L=40, T=11, u_{\mathrm{e}}(0)=0$ and $u_{\mathrm{T}}(2 L)=0$. The phenomenological FitzHugh-Nagumo model [20, 35] is used as ionic model:

$$
\begin{aligned}
I_{\text {ion }}\left(V_{\mathrm{m}}, w\right) & =w+\frac{V_{\mathrm{m}}^{3}}{3}-V_{\mathrm{m}}, \\
g\left(V_{\mathrm{m}}, w\right) & =\epsilon\left(\gamma v-V_{\mathrm{m}}-\beta\right),
\end{aligned}
$$

with $\epsilon=0.1, \beta=1$ and $\gamma=0.5$. The remaining parameters are

$$
A_{\mathrm{m}}=\epsilon^{-1}, \quad C_{\mathrm{m}}=\epsilon, \quad \sigma_{\mathrm{i}}=\sigma_{\mathrm{e}}=1, \quad \sigma_{\mathrm{T}}(x)=x / L \quad \forall x \in \Omega_{\mathrm{T}},
$$

and the initial condition is chosen as

$$
\left(V_{\mathrm{m}}^{0}, w^{0}\right)=\left\{\begin{array}{rcc}
-(1.2879,0.5758) & \text { in } & {[0,3.5),} \\
2 & \text { in } & {[3.5, L] .}
\end{array}\right.
$$

$\mathrm{RR} \mathrm{n}^{\circ} 7022$ 
The corresponding solution involves the propagating wave fronts depicted in Figure 2, Continuous $\mathbb{P}_{1}$ Lagrange finite elements are used for the discretization in space and we take $\gamma=0.1$ in 4.39 - 4.40.
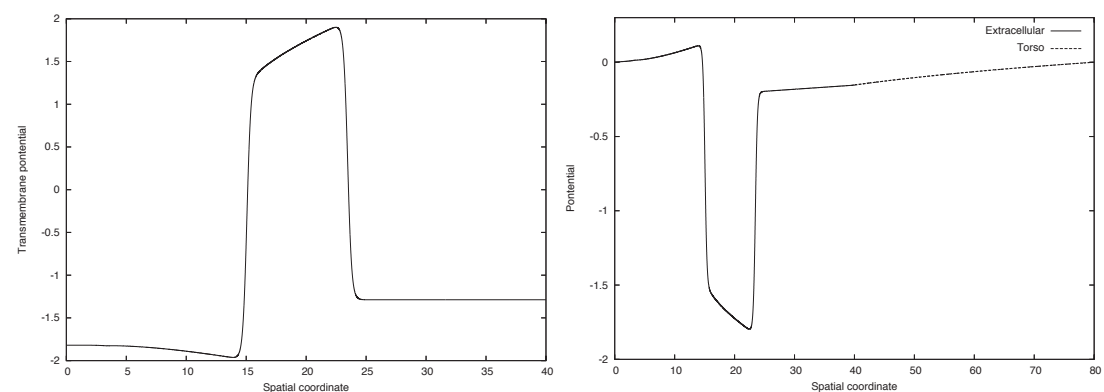

Figure 2: Transmembrane potential at time $T=11$ obtained with the isolated bidomain model (left). Heart and torso potentials at the same time instant obtained with the full bidomain (heart-torso) model (right).

\subsubsection{Isolated heart}

The isolated bidomain equations (2.1)- 2.6) are here approximated in time using the schemes 3.20$)-3.22$. For comparison purposes, we shall refer to each of these schemes with the following terminology:

- Coupled: $\left(u_{\mathrm{e}}^{\star}, V_{\mathrm{m}}^{\star}\right)=\left(u_{\mathrm{e}}^{n+1}, V_{\mathrm{m}}^{n+1}\right)$;

- Gauss-Seidel: $\left(u_{\mathrm{e}}^{\star}, V_{\mathrm{m}}^{\star}\right)=\left(u_{\mathrm{e}}^{n}, V_{\mathrm{m}}^{n+1}\right)$;

- Jacobi: $\left(u_{\mathrm{e}}^{\star}, V_{\mathrm{m}}^{\star}\right)=\left(u_{\mathrm{e}}^{n}, V_{\mathrm{m}}^{n}\right)$.

In order to illustrate the time convergence behavior (i.e. with respect to $\tau$ ) of these schemes, we have reported in Figure 3 the transmembrane potential error, at final time $T,\left\|V_{\mathrm{m}}^{\mathrm{ref}}-V_{\mathrm{m}}^{N}\right\|_{0, \Omega_{\mathrm{H}}}$, for different values of $\tau$. A fine enough space resolution, $h=2 \times 10^{-3}$, has been used in order to limit the impact of the space discretization error in the convergence history. The reference numerical solution $V_{\mathrm{m}}^{\text {ref }}$ has been generated with the Coupled scheme and a very small time-step size, $\tau=10^{-4}$.

Figure 3 shows that the Coupled, the Gauss-Seidel and the Jacobi timemarching schemes all provide the expected first order accuracy $O(\tau)$ in time. Therefore, the considered electro-diffusive splittings do not compromise the time convergence rate. Note that, at a given time-step size, Gauss-Seidel is slightly more accurate than Jacobi and Coupled than Gauss-Seidel. Somehow, this accuracy shifting is in agreement with the energy-norm weakening observed in the stability analysis of section $\$ 3$ (see Remark 3.3).

\subsubsection{Heart-torso coupling}

We now investigate the convergence behavior of the time-marching schemes introduced in section 4 . We shall refer to each of these schemes using the following terminology: 


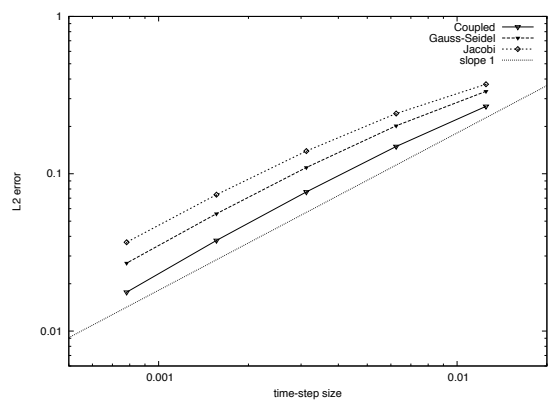

Figure 3: Time convergence history of the transmembrane potential error for the Coupled, Gauss-Seidel and Jacobi bidomain time-marching schemes (fixed space grid, $h=2 \times 10^{-3}$ ).

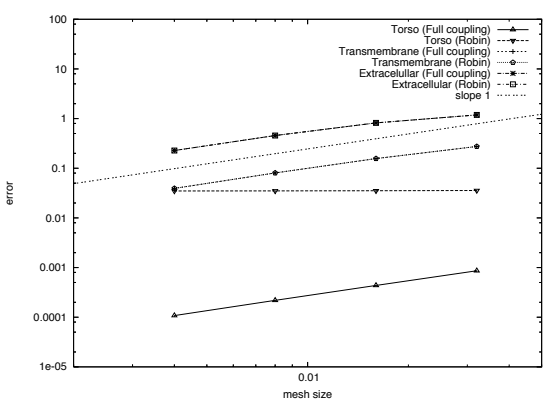

(a) Full coupling and Robin schemes, $\tau=$ $O(h)$.

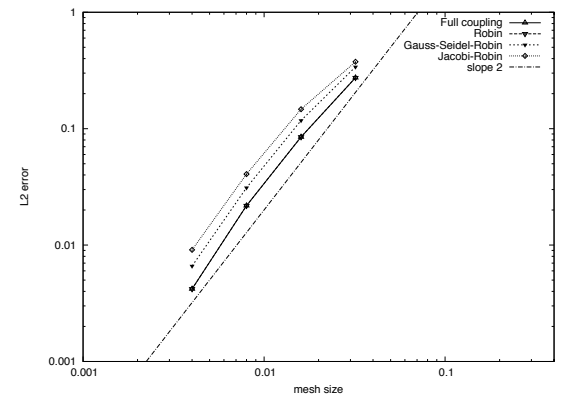

(c) Transmembrane potential error, $\tau=($ d) Extracellular potential error, $\tau=$ $O\left(h^{2}\right)$.

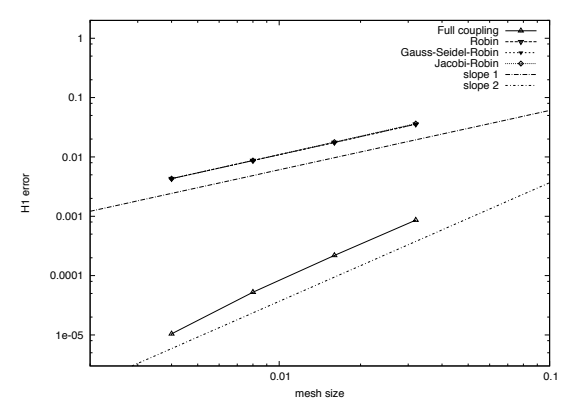

(b) Torso potential error, $\tau=O\left(h^{2}\right)$.

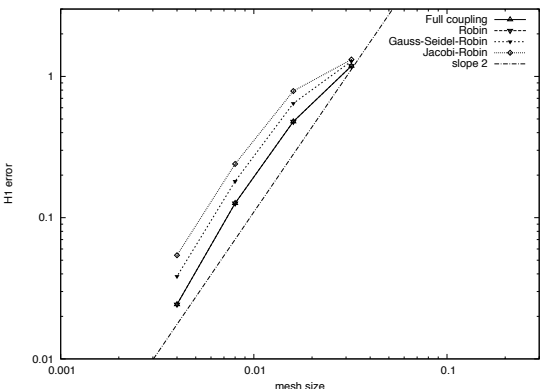

Figure 4: Convergence behavior of the different heart-torso time marching schemes.

- Full coupling: $\left(u_{\mathrm{e}}^{\star}, V_{\mathrm{m}}^{\star}\right)=\left(u_{\mathrm{e}}^{n+1}, V_{\mathrm{m}}^{n+1}\right)$ in 4.31-4.33);

- Robin: $\left(u_{\mathrm{e}}^{\star}, V_{\mathrm{m}}^{\star}\right)=\left(u_{\mathrm{e}}^{n+1}, V_{\mathrm{m}}^{n+1}\right)$ in 4.37)-4.40;

- Gauss-Seidel-Robin: $\left(u_{\mathrm{e}}^{\star}, V_{\mathrm{m}}^{\star}\right)=\left(u_{\mathrm{e}}^{n}, V_{\mathrm{m}}^{n+1}\right)$ in 4.37)-4.40;

- Jacobi-Robin: $\left(u_{\mathrm{e}}^{\star}, V_{\mathrm{m}}^{\star}\right)=\left(u_{\mathrm{e}}^{n}, V_{\mathrm{m}}^{n}\right)$ in 4.37)-4.40; 
For the convergence study, a reference heart-torso numerical solution has been generated with the Full coupling scheme and a fine space-time resolution, $\tau=$ $5 \times 10^{-5}$ and $h=2 \times 10^{-3}$.

The Full coupling scheme is expected to yield an overall $O(h+\tau)$ convergence rate, irrespectively of $h$ and $\tau$. However, for the Robin schemes 4.37)-4.40, the penalty $1 / h$ involved in the explicit Robin treatment introduces a nonstandard coupling between space and time discretizations. Indeed, a formal truncation analysis shows that the leading consistency order is $O(\tau / h)$. As a result, the hyperbolic-CFL stability condition $\tau=O(h)$ of Theorem 4.4 might not be enough to ensure convergence. This behavior is illustrated numerically in Figure 4(a), where we have reported the following errors, at time $T, \| \sigma_{\mathrm{T}} \nabla\left(u_{\mathrm{T}}^{\mathrm{ref}}-\right.$ $\left.u_{\mathrm{T}}^{N}\right)\left\|_{0, \Omega_{\mathrm{T}}},\right\| \sigma_{\mathrm{e}} \nabla\left(u_{\mathrm{e}}^{\mathrm{ref}}-u_{\mathrm{e}}^{N}\right)\left\|_{0, \Omega_{\mathrm{H}}},\right\| V_{\mathrm{m}}^{\mathrm{ref}}-V_{\mathrm{m}}^{N} \|_{0, \Omega_{\mathrm{H}}}$ for the Full coupling and Robin schemes, in terms of $h$ with $\tau / h=2.56$. As indicated above, the torso error remains constant for the Robin scheme. Instead, we can take $\tau=O\left(h^{2}\right)$ to obtain an overall consistency of $O(h)$, which can be considered optimal for piecewise affine space approximations. This is illustrated in Figure 4(b) Robin, Gauss-Seidel-Robin and Jacobi-Robin all yield the expected first order torso accuracy $O(h)$ with $\tau / h^{2} \approx 12$. Note that, in this case, the Full coupling scheme provides a superior accuracy $O\left(h^{2}\right)$. This suggests that, for the considered set of discretization parameters, the error is mainly driven by the time discretization. In Figures 4(c) and 4(d) (and also in Figure 4(a) we can observe that all the considered methods provide practically the same accuracy on the heart potentials $V_{\mathrm{m}}$ and $u_{\mathrm{e}}$.

In summary, the superior stability properties (compared to Dirichlet-Neumann) and computational cost reduction (compared to Full coupling) featured by the proposed Robin heart-torso decoupling schemes come with a price: a condition $\tau=O\left(h^{2}\right)$ is required to guarantee an overall $O(h)$ convergence rate. We shall see, in the tree-dimensional study of the next section, that these schemes are well-suited to simulate accurate ECG signals.

\subsection{Three-dimensional study}

Results for the isolated bidomain model using the time-marching procedures of section $\$ 3$ are presented in subsection $\$ 5.2 .2$. Subsection $\$ 5.2 .3$ demonstrates the capabilities of the splitting schemes introduced in section $\$ 4$ to provide accurate 12-lead ECG signals.

\subsubsection{Simulation data}

The simulations are performed with the anatomical data and the model parameters used in 11, 12. For the sake of conciseness we only report here the main ingredients (full details are given in [11, 12]).

The computational heart and torso meshes were obtained starting from the Zygote (www.3dscience.com) anatomical model, using the 3-matic software (www.materialise.com) to obtain computationally-correct surface meshes. The mesh, displayed in Figure 5, contains the heart, lung, bones and remaining extramyocardial tissue. They have been obtained by processing the surface meshes with Yams 21] and GHS3D 22. The volume heart and torso meshes are made of 542000 and 1242000 tetrahedra, respectively. 


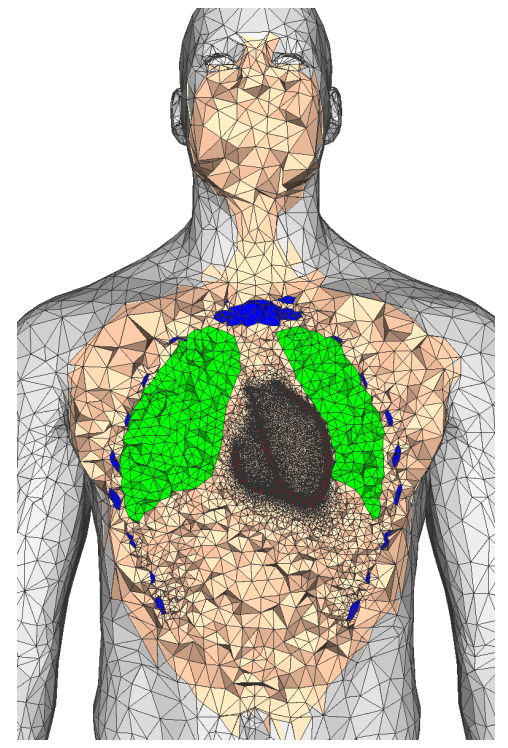

Figure 5: Cut view of the heart-torso computational mesh: heart (red) lungs (green), bone (blue) and remaining tissue (apricot).

The bidomain model parameters are given in Table 1] where $\sigma_{\mathrm{i}, \mathrm{e}}^{\mathrm{l}}\left(\right.$ resp. $\left.\sigma_{\mathrm{i}, \mathrm{e}}^{\mathrm{t}}\right)$ denotes the scalar intra- and extracelluar longitudinal (resp. transverse) conductivities. Table 2 provides the conductivity parameters for the torso (supposed isotropic).

\begin{tabular}{|c|c|c|c|c|c|}
\hline$A_{\mathrm{m}}\left(\mathrm{cm}^{-1}\right)$ & $C_{\mathrm{m}}(\mathrm{mF})$ & $\sigma_{\mathrm{i}}^{\perp}\left(\mathrm{S} \mathrm{cm}^{-1}\right)$ & $\sigma_{\mathrm{e}}^{\mathrm{I}}\left(\mathrm{S} \mathrm{cm}^{-1}\right)$ & $\sigma_{\mathrm{i}}^{\mathrm{t}}\left(\mathrm{S} \mathrm{cm}^{-1}\right)$ & $\sigma_{\mathrm{e}}^{\mathrm{t}}\left(\mathrm{S} \mathrm{cm}^{-1}\right)$ \\
\hline 500 & $10^{-3}$ & $3.0 \times 10^{-3}$ & $3.0 \times 10^{-3}$ & $3.0 \times 10^{-4}$ & $1.2 \times 10^{-3}$ \\
\hline
\end{tabular}

Table 1: Bidomain model parameters.

\begin{tabular}{|c|c|c|}
\hline$\sigma_{\mathrm{T}}^{\mathrm{t}}\left(\mathrm{S} \mathrm{cm}^{-1}\right)$ & $\sigma_{\mathrm{T}}^{\mathrm{I}}\left(\mathrm{S} \mathrm{cm}^{-1}\right)$ & $\sigma_{\mathrm{T}}^{\mathrm{b}}\left(\mathrm{S} \mathrm{cm}^{-1}\right)$ \\
\hline $6.0 \times 10^{-4}$ & $2.4 \times 10^{-4}$ & $4.0 \times 10^{-5}$ \\
\hline
\end{tabular}

Table 2: Torso conductivity parameters: tissue (t), lungs (l) and bone (b).

As in 4, a rescaled version of the phenomenological two-variable model proposed by Mitchell and Schaeffer in 33] is considered as ionic model. Functions $g$ and $I_{\text {ion }}$ are then given by

$$
\begin{gathered}
I_{\text {ion }}\left(V_{\mathrm{m}}, w\right)=-w \frac{\left(V_{\mathrm{m}}-V_{\min }\right)^{2}\left(V_{\max }-V_{\mathrm{m}}\right)}{\tau_{\mathrm{in}}\left(V_{\max }-V_{\min }\right)}+\frac{V_{\mathrm{m}}-V_{\min }}{\tau_{\text {out }}\left(V_{\max }-V_{\min }\right)}, \\
g\left(V_{\mathrm{m}}, w\right)=\left\{\begin{aligned}
\frac{w}{\tau_{\text {open }}}-\frac{1}{\tau_{\text {open }}\left(V_{\max }-V_{\min }\right)^{2}} & \text { if } V_{\mathrm{m}}<V_{\text {gate }}, \\
\frac{w}{\tau_{\text {close }}} & \text { if } \quad V_{\mathrm{m}} \geq V_{\text {gate }},
\end{aligned}\right.
\end{gathered}
$$


where the values of the free parameters $\tau_{\text {in }}, \tau_{\text {out }}, \tau_{\text {open }}, \tau_{\text {close }}, V_{\text {gate }}$ are reported in Table 3 and $V_{\min }, V_{\max }$ are scaling constants $(-80$ and $20 \mathrm{mV}$, respectively).

\begin{tabular}{|c|c|c|c|c|c|c|}
\hline$\tau_{\text {in }}$ & $\tau_{\text {out }}$ & $\tau_{\text {open }}$ & $\tau_{\text {close }}^{\mathrm{RV}}$ & $\tau_{\text {close }}^{\mathrm{LV}-\text { endo }}$ & $\tau_{\text {close }}^{\mathrm{LV}-\text { epi }}$ & $V_{\text {gate }}$ \\
\hline 4.5 & 90 & 100 & 120 & 140 & 105 & -67 \\
\hline
\end{tabular}

Table 3: Mitchell-Schaeffer ionic model parameters.

Continuous $\mathbb{P}_{1}$ Lagrange finite elements are used for the space discretization of both the heart and the torso equations. The time step size was fixed to $\tau=0.25 \mathrm{~ms}$ and the Robin parameter, for the heart-torso coupling scheme 4.37 -4.40, to $\gamma=0.1$.

\subsubsection{Isolated heart}

The isolated bidomain equations (2.1)- 2.6 are approximated using the timemarching schemes 3.20-3.22). We shall refer to each of these schemes using the terminology of subsection $\$ 5.1 .2$.
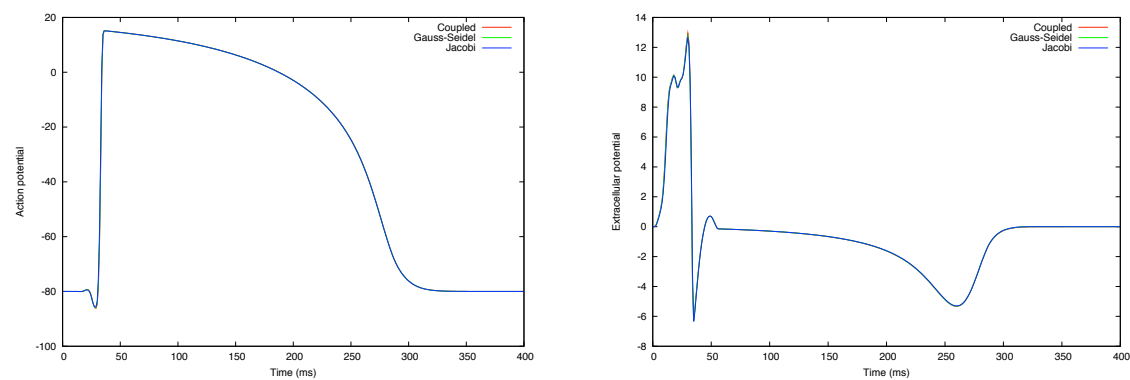

Figure 6: Time course of the transmembrane potential (left) and extracellular potential (right) at a given location in the epicardium.

The time course of the transmembrane and extracellular potentials at a given epicardial location are displayed in Figure 6. We can observe that the simulations are numerically stable and the curves are practically indistinguishable. Somehow, this is not surprising since, as shown in subsection $\$ 5.1 .2$, the electro-diffusive Gauss-Seidel and Jacobi splittings still provide optimal first order accuracy. A slight difference can be seen after axis rescaling, as shown in Figure 7 .

The results reported in Table 4 confirm that the electro-diffusive GaussSeidel and Jacobi splittings do not introduce additional constraints on the time step size $\tau$, as predicted by Theorem 3.2

\subsubsection{2-lead ECG}

The simulated 12-lead ECG signals obtained with the explicit Robin coupling procedures 4.37)-4.40 are here compared with those obtained using full hearttorso coupling (see e.g. [30, 4]) and heart-torso uncoupling (see e.g. [4] and 

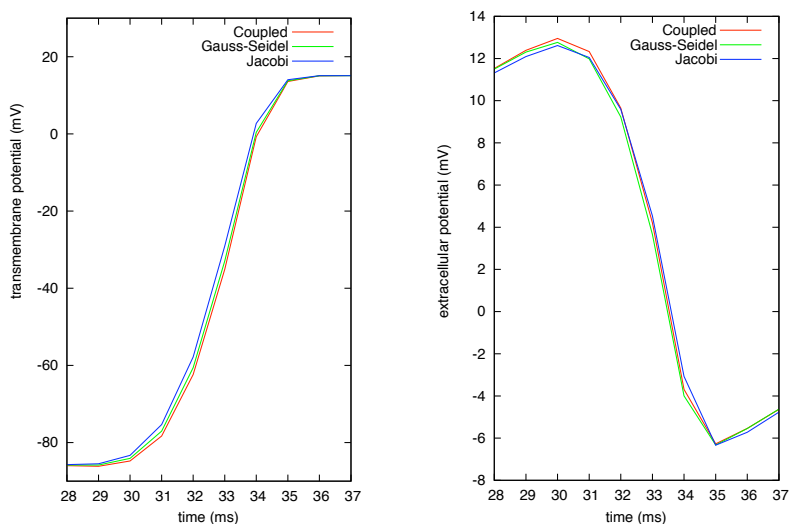

Figure 7: Time course, after axis rescaling, of the transmembrane potential (left) and extracellular potential (right) at a given location in the epicardium.

\begin{tabular}{|c|c|c|c|}
\hline$\tau_{\tau(\mathrm{ms})}$ scheme & Coupled & Gauss-Seidel & Jacobi \\
\hline 0.25 & $\sqrt{ }$ & $\bar{\checkmark}$ & $\bar{\top}$ \\
\hline 0.50 & $\sqrt{ }$ & $\checkmark$ & $\checkmark$ \\
\hline 1.00 & $\sqrt{ }$ & $\checkmark$ & $\checkmark$ \\
\hline 1.25 & $x$ & $x$ & $x$ \\
\hline 1.50 & $x$ & $x$ & $x$ \\
\hline
\end{tabular}

Table 4: Stability sensitivity to the time step size $\tau$. Symbol $\times$ indicates $n u-$ merical instability.

Remark 2.2. For comparison purposes, the latter will be termed as Uncoupling and, for the rest, we will employ the terminology used in subsection 5.1 .3 .

In Figures 8 and 9 we provide the complete 12-lead ECG signals obtained using the full coupling (black) and uncoupling (red) approaches. We can observe that the uncoupling approach is unable to reproduce the correct signal amplitude, which is indeed magnified by a factor close to 2 in practically all the ECG leads. Moreover, the shape mismatch in some of the leads is clearly visible: the QRS complex in V3 and the T-wave in V2, for instance. Similar observations have been reported in [4] (see also [30]), for both healthy and pathological conditions.

In the next paragraphs, we shall see that (for an equivalent computational cost) the Robin based explicit coupling introduced in section $\$ 4$ provides much more accurate ECG signals. For illustration purposes, in Figure 13 , we have reported some snapshots of the body surface potentials obtained with the JacobiRobin scheme. Figure 12 shows a posterior view of the potential within the torso and the heart. The potential matching at the heart-torso interface is clearly visible.

In Figures 10 and 11 we compare the simulated 12-lead ECG signals obtained with full coupling (black) to those obtained with fully decoupled Jacobi-Robin scheme (red). The improved accuracy with respect to the uncoupling approach is striking. Indeed, the signals are practically indistinguishable in all the 12 

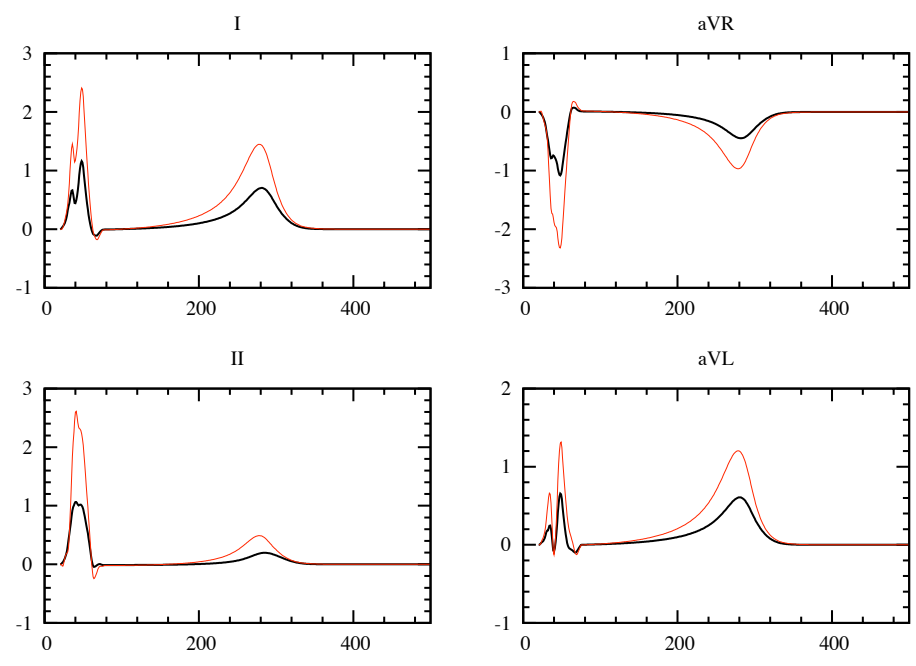

III
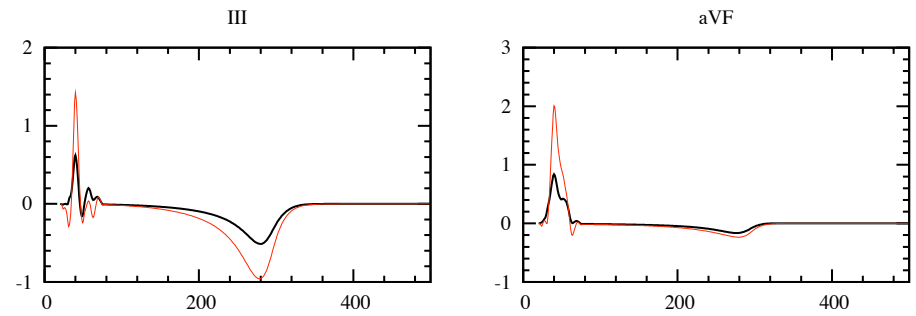

Figure 8: Simulated ECG signals (standard and augmented leads) obtained using heart-torso full coupling (black) and uncoupling (red).

leads. Some minor differences are visible in the QRS complex of V2 and V3. Similar results are obtained with the Robin and Gauss-Seidel-Robin schemes, that we omit for the sake of conciseness. Nevertheless, in order to illustrate the impact of the level of decoupling in the accuracy of the ECG, we have reported in Figure 14 a rescaled comparison of the QRS complex (left) and T-wave (right) of the first ECG lead, obtained with the full coupling, Robin, Gauss-Seidel-Robin and Jacobin-Robin approaches. No significant differences are observed in the T-wave, whereas slightly better results are obtained with the Robin approach in the QRS-complex.

The 12-lead ECG signals of a pathological situation, a left bundle branch block (LBBB), have been also computed to illustrate the robustness of the proposed splitting schemes. Figures 15 and 16 presents the corresponding signals obtained with the full coupling (black) and Jacobi-Robin (red) schemes. Once more, the decoupled scheme shows very good accuracy and stability.

Finally, we go further in the investigation of the robustness of the schemes, by considering different heart and torso geometries and model parameters. In particular, we keep $\gamma=0.1$ as in the previous cases. To this aim, we revisit the ECG numerical simulations recently reported in [4. Figures 17 and 18 the corresponding signals obtained with the full coupling (black) and Jacobi-Robin (red) schemes. Once more, both signals are in excellent agreement. Similar 

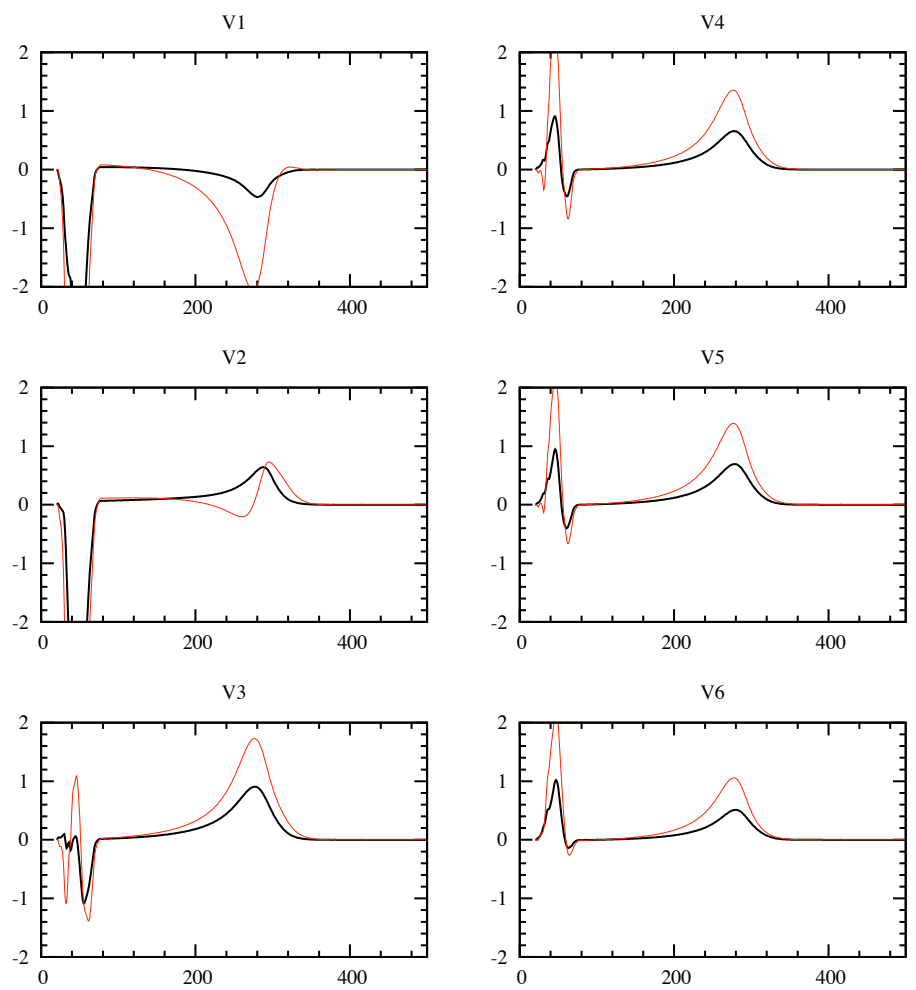

Figure 9: Simulated ECG signals (chest leads) obtained using heart-torso full coupling (black) and uncoupling (red).

results have been obtained for a LBBB pathology, that we omit here for the sake of conciseness.

\section{Conclusion}

We have introduced and analyzed a series of first order semi-implicit timemarching schemes for the cardiac bidomain equations, either isolated or coupled with generalized Laplace equation for the torso. The main feature of the analyzed schemes is that they all allow a fully decoupled computation of the ionic state, the transmembrane potential, the extracellular potential and the torso potential.

For the isolated bidomain model, Theorem 3.2 shows that the Gauss-Seidel and Jacobi splittings do not compromise the stability of the resulting schemes; they simply alter the energy norm. Moreover, the time step restrictions are only dictated by the semi-implicit treatment of then non-linear reaction terms. The numerical results, reported in subsections $\$ 5.1 .2$ and $\$ 5.2 .2$. confirmed these theoretical findings and demonstrated that the splitting preserves the first-order time accuracy $O(\tau)$ of the original electro-diffusive coupling.

We extended these time-marching techniques to the numerical simulation of the ECG, by combining the Gauss-Seidel and the Jacobi like bidomain spplitings 

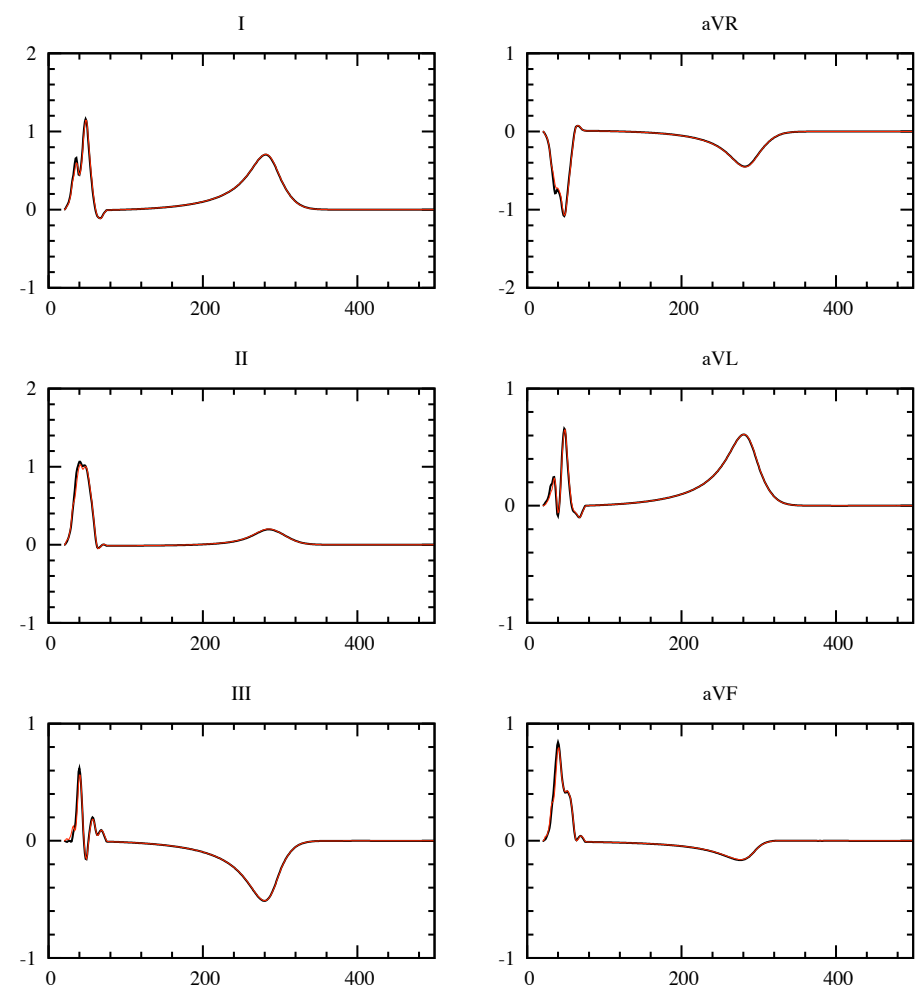

Figure 10: Simulated ECG signals (standard and augmented leads) obtained using heart-torso full coupling (black) and the Jacobi-Robin scheme (red).

with an explicit Robin-Robin heart-torso coupling. This specific treatment of the heat-torso coupling is well-suited, particularly, since the time discretization of the two (quasi-static) elliptic equations does not produce numerical dissipation and, therefore, conventional Dirichlet-Neumann explicit coupling might lead to numerical instability. Theorem 4.4 shows that the proposed splitting schemes are stable under an additional hyperbolic-CFL condition $\tau=O(h)$. As regards the convergence behavior, the numerical study of subsection $\$ 5.1 .3$ showed that a condition $\tau=O\left(h^{2}\right)$ is required to guarantee an overall asymptotic rate of $O(h)$ (optimal for piecewise affine approximations in space). This limitation comes from the semi-implicit treatment of the stabilizing penalty term scaling with $1 / h$. In spite of that, the numerical study reported in section 5.2.3 using anatomical heart and torso geometries, demonstrated that the Gauss-Seidel-Robin and the Jacobi-Robin splittings are able to provide accurate 12-lead ECG signals, both for a healthy and a pathological condition. Note that this is a major advantage with respect to the conventional heart-torso uncoupling approximation, which (for a similar computational cost) is known to provide inaccurate ECG signals (see e.g. 30, 40, 4). Somehow the discretization error introduced by the Robin heart-torso decoupling is negligible with respect to the modeling error involved in the heart-torso uncoupling approximation. The robustness of the proposed splitting schemes has been also 

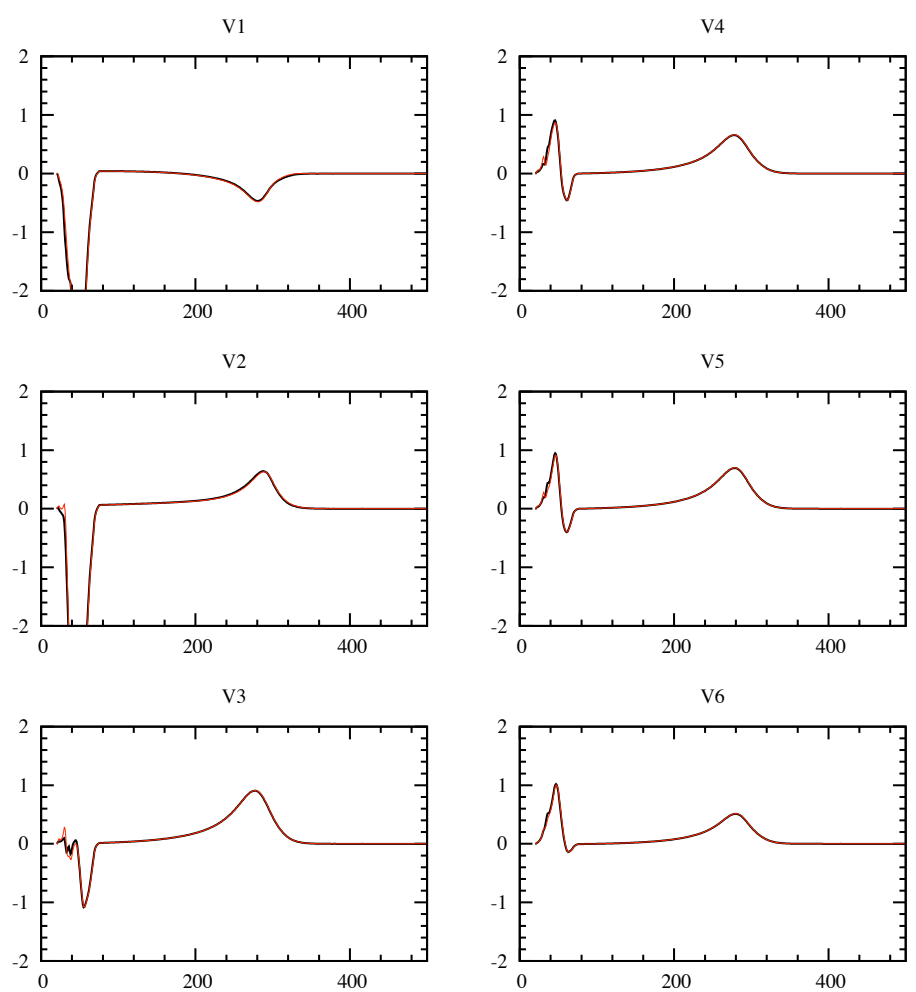

Figure 11: Simulated ECG signals (chest leads) obtained using heart-torso full coupling (black) and the Jacobi-Robin scheme (red).

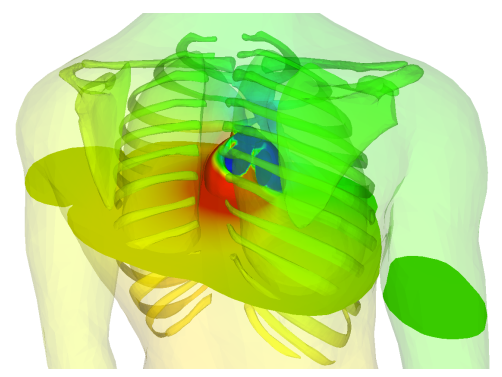

Figure 12: Posterior view and cut plane of the torso and heart potentials at time $t=10 \mathrm{~ms}$

illustrated with numerical experiments based on different model parameters and heart/torso geometries.

The theoretical and numerical study of this paper is limited to discretizations yielding first order accuracy in time. Some insights into feasible extensions to higher order are commented in Remark 3.4. Although the present stability analysis holds irrespectively of the original time discretization scheme, it does depend on the (first order) extrapolation involved in the splittings. As a result, the generalization of the present analysis to hight order extrapolations seems 


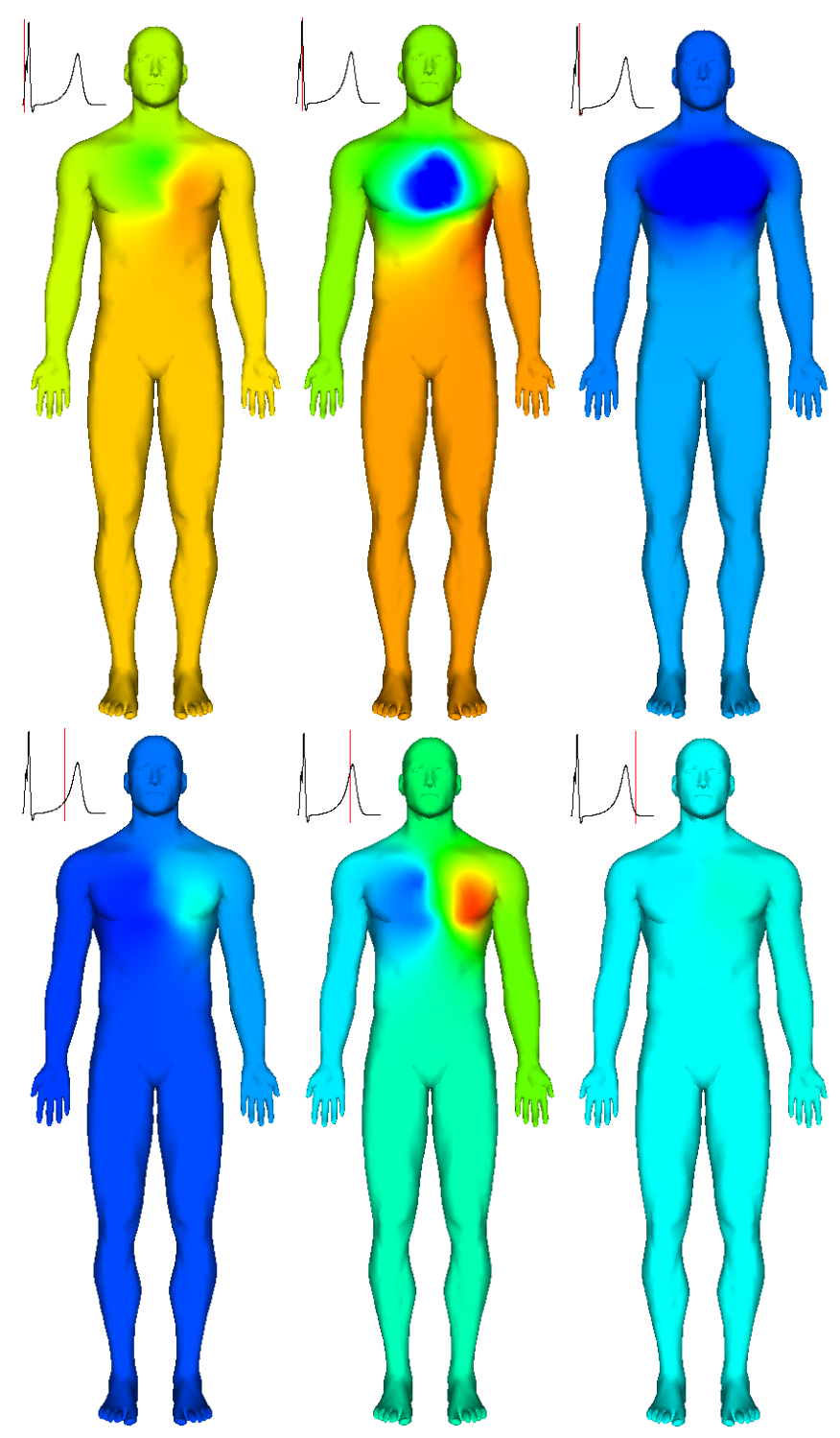

Figure 13: Snapshots of the body surface potentials at times $t=10,32,40$, 200, 250 and $310 \mathrm{~ms}$ (from left to right and top to bottom).

not straightforward. Further numerical investigations would certainly help to clarify this issue and could be the topic of future work.

\section{Acknowledgements}

This work was partially supported by INRIA through its large scope initiative CardioSense3D (www.inria.fr/CardioSense3D). We wish to thank JeanFrédéric Gerbeau and Muriel Boulakia for many fruitful discussions on ECG modeling and simulation. We also thank Philippe Moireau and Elsie Phé for 

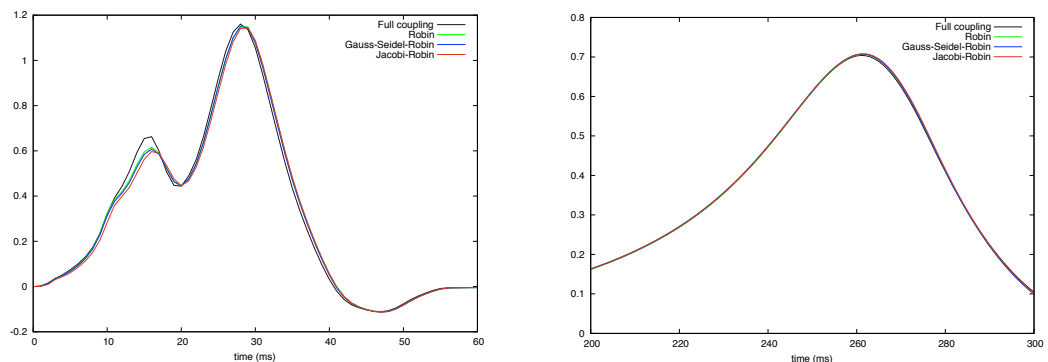

Figure 14: Comparison of the QRS complex (left) and T-wave (right) of the first ECG lead: Full coupling (black), Robin (green), Gauss-Seidel-Robin (blue), Jacobin-Robin (red).
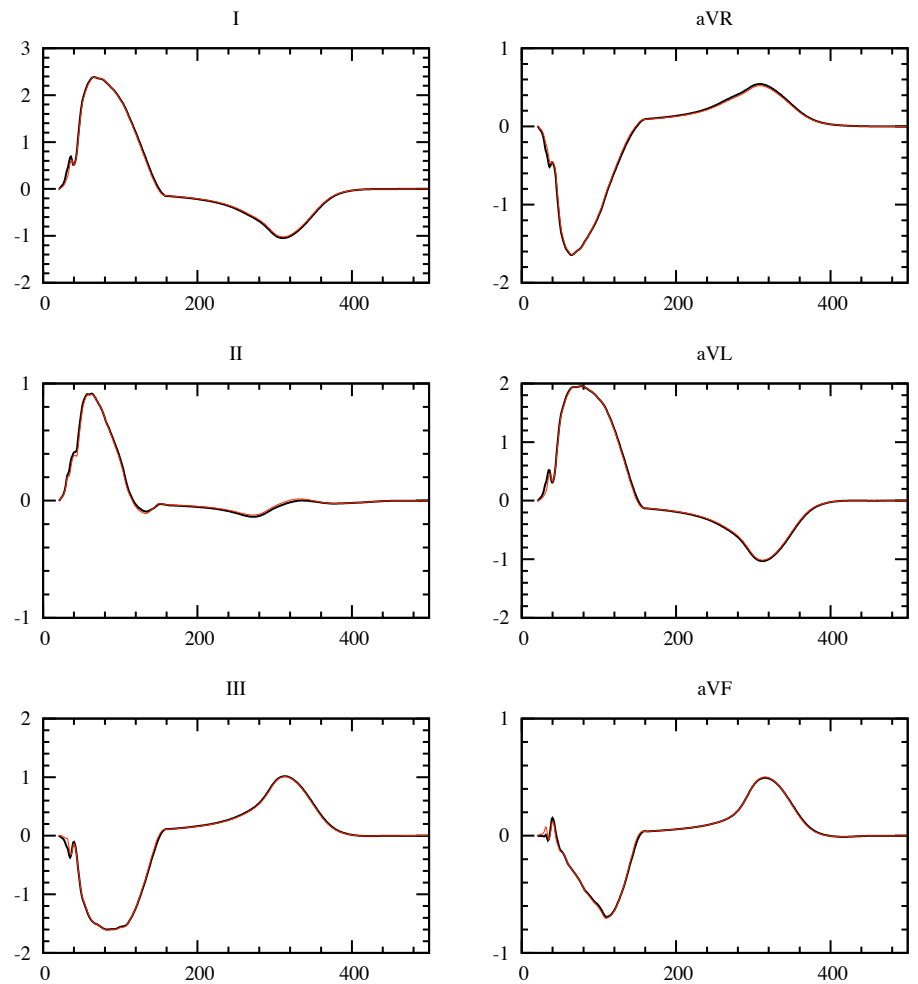

Figure 15: Simulated ECG signals (standard and augmented leads) for a LBBB pathology, obtained using heart-torso full coupling (black) and the Jacobi-Robin scheme (red).

their work on the anatomical models and meshes. The anonymous referees are acknowledged for their comments that helped to improve the manuscript. 

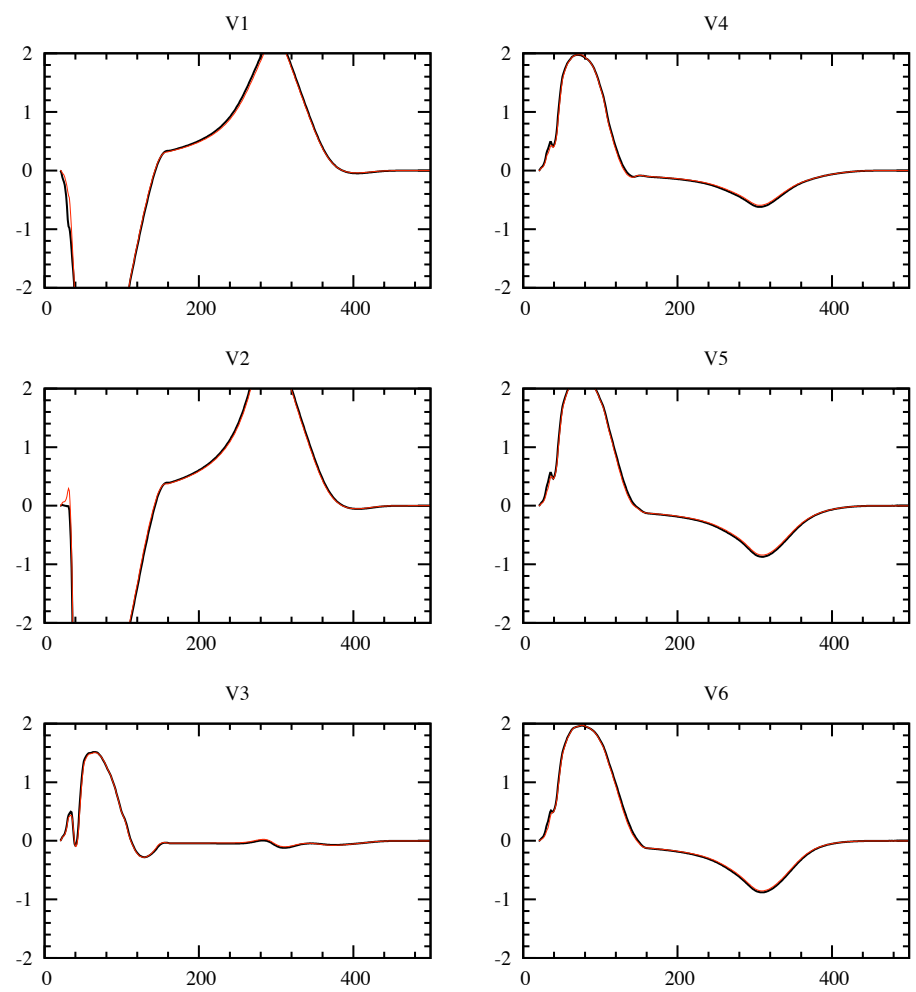

Figure 16: Simulated ECG signals (chest leads) for a LBBB pathology, obtained using heart-torso full coupling (black) and the Jacobi-Robin scheme (red).

\section{A Proofs of the stability results}

\section{A.1 Proof of Theorem 3.2}

Under assumptions 3.23 and $(3.24)$, the stability estimate 3.25$)$, for the monolithic case $\left(u_{\mathrm{e}}^{\star}, V_{\mathrm{m}}^{\star}\right)=\left(u_{\mathrm{e}}^{n+1}, V_{\mathrm{m}}^{n+1}\right)$, can be straightforwardly derived from the analysis reported in [19] (see also [47]). Therefore, we only detail here the proofs of (3.26) and (3.27).

Let first consider the Gauss-Seidel like decoupling $\left(u_{\mathrm{e}}^{\star}, V_{\mathrm{m}}^{\star}\right)=\left(u_{\mathrm{e}}^{n}, V_{\mathrm{m}}^{n+1}\right)$. By testing 3.20)-(3.22 with $\tau\left(w^{n+1}, V_{\mathrm{m}}^{n+1}, u_{\mathrm{e}}^{n+1}\right)$ and after summation of the 

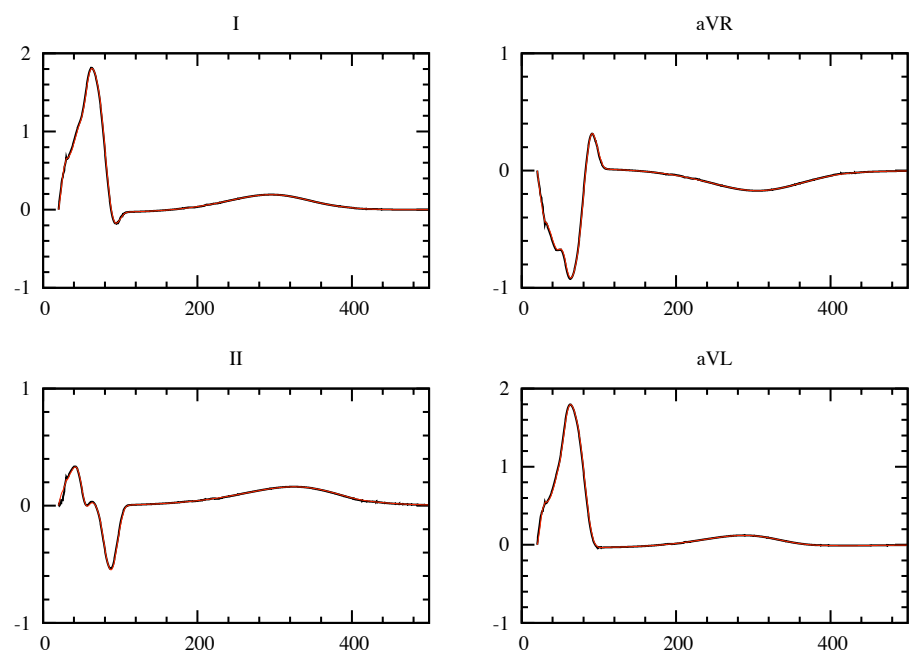

III

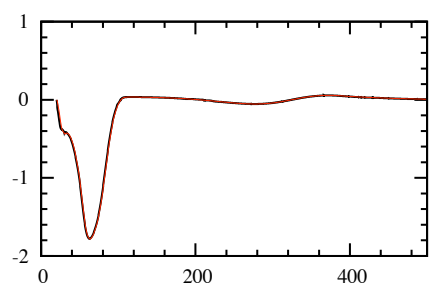

aVF

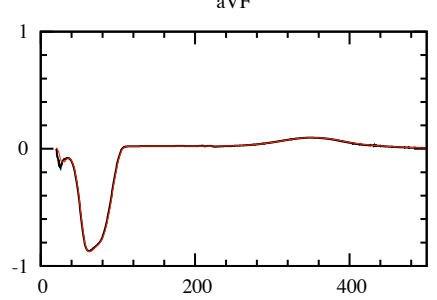

Figure 17: Simulated ECG signals (standard and augmented leads) obtained using heart-torso full coupling (black) and the Jacobi-Robin scheme (red). Geometry data and model parameters from [4].

resulting expressions we have:

$$
\begin{gathered}
\frac{1}{2}\left(\left\|w^{n+1}\right\|_{0, \Omega_{\mathrm{H}}}^{2}-\left\|w^{n}\right\|_{0, \Omega_{\mathrm{H}}}^{2}\right)+\frac{\chi_{\mathrm{m}}}{2}\left(\left\|V_{\mathrm{m}}^{n+1}\right\|_{0, \Omega_{\mathrm{H}}}^{2}-\left\|V_{\mathrm{m}}^{n}\right\|_{0, \Omega_{\mathrm{H}}}^{2}\right)+\tau\left\|\boldsymbol{\sigma}_{\mathrm{e}}^{\frac{1}{2}} \boldsymbol{\nabla} u_{\mathrm{e}}^{n+1}\right\|_{0, \Omega_{\mathrm{H}}}^{2} \\
+\tau\left\|\boldsymbol{\sigma}_{\mathrm{i}}^{\frac{1}{2}} \nabla\left(V_{\mathrm{m}}^{n+1}+u_{\mathrm{e}}^{n+1}\right)\right\|_{0, \Omega_{\mathrm{H}}}^{2}+\underbrace{\tau \int_{\Omega_{\mathrm{H}}} \boldsymbol{\sigma}_{\mathrm{i}} \boldsymbol{\nabla}\left(u_{\mathrm{e}}^{n}-u_{\mathrm{e}}^{n+1}\right) \cdot \nabla V_{\mathrm{m}}^{n+1} \mathrm{~d} \boldsymbol{x}}_{I_{1}} \\
\leq \underbrace{\tau \int_{\Omega_{\mathrm{H}}}\left(I_{\mathrm{app}}^{n+1}-I_{\mathrm{ion}}\left(V_{\mathrm{m}}^{n}, w^{n+1}\right)\right) V_{\mathrm{m}}^{n+1} \mathrm{~d} \boldsymbol{x}-\tau \int_{\Omega_{\mathrm{H}}} g\left(V_{\mathrm{m}}^{n}, w^{n+1}\right) w^{n+1} \mathrm{~d} \boldsymbol{x}}_{I_{2}},
\end{gathered}
$$

where the square roots $\boldsymbol{\sigma}_{\mathrm{e}}^{\frac{1}{2}}, \boldsymbol{\sigma}_{\mathrm{i}}^{\frac{1}{2}}$ are well defined since $\boldsymbol{\sigma}_{\mathrm{i}}, \boldsymbol{\sigma}_{\mathrm{e}}$ are symmetric and positive-definite tensors (see e.g. [42]). We now provide appropriate bounds for terms $I_{1}$ and $I_{2}$. 



Figure 18: Simulated ECG signals (chest leads) obtained using heart-torso full coupling (black) and the Jacobi-Robin scheme (red). Geometry data and model parameters from [4].

As regards the first term, we have

$$
\begin{aligned}
I_{1}= & \tau \int_{\Omega_{\mathrm{H}}} \boldsymbol{\sigma}_{\mathrm{i}} \boldsymbol{\nabla}\left(u_{\mathrm{e}}^{n}-u_{\mathrm{e}}^{n+1}\right) \cdot \boldsymbol{\nabla}\left(V_{\mathrm{m}}^{n+1}+u_{\mathrm{e}}^{n+1}\right) \mathrm{d} \boldsymbol{x}+\int_{\Omega_{\mathrm{H}}} \boldsymbol{\sigma}_{\mathrm{i}} \boldsymbol{\nabla}\left(u_{\mathrm{e}}^{n+1}-u_{\mathrm{e}}^{n}\right) \cdot \boldsymbol{\nabla} u_{\mathrm{e}}^{n+1} \mathrm{~d} \boldsymbol{x} \\
\geq & -\frac{\tau}{2}\left\|\boldsymbol{\sigma}_{\mathrm{i}}^{\frac{1}{2}} \nabla\left(u_{\mathrm{e}}^{n}-u_{\mathrm{e}}^{n+1}\right)\right\|_{0, \Omega_{\mathrm{H}}}^{2}-\frac{\tau}{2}\left\|\boldsymbol{\sigma}_{\mathrm{i}}^{\frac{1}{2}} \nabla\left(V_{\mathrm{m}}^{n+1}+u_{\mathrm{e}}^{n+1}\right)\right\|_{0, \Omega_{\mathrm{H}}}^{2} \\
& +\frac{\tau}{2}\left(\left\|\boldsymbol{\sigma}_{\mathrm{i}}^{\frac{1}{2}} \nabla u_{\mathrm{e}}^{n+1}\right\|_{0, \Omega_{\mathrm{H}}}^{2}-\left\|\boldsymbol{\sigma}_{\mathrm{i}}^{\frac{1}{2}} \boldsymbol{\nabla} u_{\mathrm{e}}^{n}\right\|_{0, \Omega_{\mathrm{H}}}^{2}\right)+\frac{\tau}{2}\left\|\boldsymbol{\sigma}_{\mathrm{i}}^{\frac{1}{2}} \boldsymbol{\nabla}\left(u_{\mathrm{e}}^{n+1}-u_{\mathrm{e}}^{n}\right)\right\|_{0, \Omega_{\mathrm{H}}}^{2} \\
= & \frac{\tau}{2}\left(\left\|\boldsymbol{\sigma}_{\mathrm{i}}^{\frac{1}{2}} \nabla u_{\mathrm{e}}^{n+1}\right\|_{0, \Omega_{\mathrm{H}}}^{2}-\left\|\boldsymbol{\sigma}_{\mathrm{i}}^{\frac{1}{2}} \nabla u_{\mathrm{e}}^{n}\right\|_{0, \Omega_{\mathrm{H}}}^{2}\right)-\frac{\tau}{2}\left\|\boldsymbol{\sigma}_{\mathrm{i}}^{\frac{1}{2}} \nabla\left(V_{\mathrm{m}}^{n+1}+u_{\mathrm{e}}^{n+1}\right)\right\|_{0, \Omega_{\mathrm{H}}}^{2} .
\end{aligned}
$$

On the other hand, from (3.23), for the second term can be bounded as follows

$$
\begin{aligned}
I_{2}= & \frac{\tau}{2}\left\|I_{\mathrm{app}}^{n+1}\right\|_{0, \Omega_{\mathrm{H}}}^{2}+\frac{\tau}{2}\left\|V_{\mathrm{m}}^{n+1}\right\|_{0, \Omega_{\mathrm{H}}}^{2}+\frac{\tau C_{I}}{2}\left(3\left\|V_{\mathrm{m}}^{n+1}\right\|_{0, \Omega_{\mathrm{H}}}^{2}+\left\|w^{n+1}\right\|_{0, \Omega_{\mathrm{H}}}^{2}\right) \\
& +\frac{\tau C_{g}}{2}\left(\left\|V_{\mathrm{m}}^{n+1}\right\|_{0, \Omega_{\mathrm{H}}}^{2}+3\left\|w^{n+1}\right\|_{0, \Omega_{\mathrm{H}}}^{2}\right) \\
= & \frac{\tau}{2}\left\|I_{\mathrm{app}}^{n+1}\right\|_{0, \Omega_{\mathrm{H}}}^{2}+\frac{\tau}{2} \underbrace{\left(1+3 C_{I}+C_{g}\right)}_{\alpha}\left\|V_{\mathrm{m}}^{n+1}\right\|_{0, \Omega_{\mathrm{H}}}^{2}+\frac{\tau}{2} \underbrace{\left(C_{I}+3 C_{g}\right)}_{\beta}\left\|w^{n+1}\right\|_{0, \Omega_{\mathrm{H}}}^{2} .
\end{aligned}
$$


As a result, inserting A.44 and A.45 into A.43, yields

$$
\begin{gathered}
\frac{1}{2}\left(\left\|w^{n+1}\right\|_{0, \Omega_{\mathrm{H}}}^{2}-\left\|w^{n}\right\|_{0, \Omega_{\mathrm{H}}}^{2}\right)+\frac{\chi_{\mathrm{m}}}{2}\left(\left\|V_{\mathrm{m}}^{n+1}\right\|_{0, \Omega_{\mathrm{H}}}^{2}-\left\|V_{\mathrm{m}}^{n}\right\|_{0, \Omega_{\mathrm{H}}}^{2}\right)+\tau\left\|\boldsymbol{\sigma}_{\mathrm{e}}^{\frac{1}{2}} \nabla u_{\mathrm{e}}^{n+1}\right\|_{0, \Omega_{\mathrm{H}}}^{2} \\
+\frac{\tau}{2}\left\|\boldsymbol{\sigma}_{\mathrm{i}}^{\frac{1}{2}} \nabla\left(V_{\mathrm{m}}^{n+1}+u_{\mathrm{e}}^{n+1}\right)\right\|_{0, \Omega_{\mathrm{H}}}^{2}+\frac{\tau}{2}\left(\left\|\boldsymbol{\sigma}_{\mathrm{i}}^{\frac{1}{2}} \nabla u_{\mathrm{e}}^{n+1}\right\|_{0, \Omega_{\mathrm{H}}}^{2}-\left\|\boldsymbol{\sigma}_{\mathrm{i}}^{\frac{1}{2}} \nabla u_{\mathrm{e}}^{n}\right\|_{0, \Omega_{\mathrm{H}}}^{2}\right) \\
\leq \frac{\tau}{2}\left\|I_{\mathrm{app}}^{n+1}\right\|_{0, \Omega_{\mathrm{H}}}^{2}+\frac{\tau \alpha}{2}\left\|V_{\mathrm{m}}^{n+1}\right\|_{0, \Omega_{\mathrm{H}}}^{2}+\frac{\tau \beta}{2}\left\|w^{n+1}\right\|_{0, \Omega_{\mathrm{H}}}^{2},
\end{gathered}
$$

so that, replacing index $n$ by $m$ and summing over $0 \leq m \leq n-1$, we have

$$
\begin{gathered}
\left\|w^{n}\right\|_{0, \Omega_{\mathrm{H}}}^{2}+\chi_{\mathrm{m}}\left\|V_{\mathrm{m}}^{n}\right\|_{0, \Omega_{\mathrm{H}}}^{2}+\tau\left\|\boldsymbol{\sigma}_{\mathrm{i}}^{\frac{1}{2}} \nabla u_{\mathrm{e}}^{n}\right\|_{0, \Omega_{\mathrm{H}}}^{2}+2 \sum_{m=0}^{n-1} \tau\left\|\boldsymbol{\sigma}_{\mathrm{e}}^{\frac{1}{2}} \nabla u_{\mathrm{e}}^{m+1}\right\|_{0, \Omega_{\mathrm{H}}}^{2} \\
+\sum_{m=0}^{n-1} \tau\left\|\boldsymbol{\sigma}_{\mathrm{i}}^{\frac{1}{2}} \boldsymbol{\nabla}\left(V_{\mathrm{m}}^{m+1}+u_{\mathrm{e}}^{m+1}\right)\right\|_{0, \Omega_{\mathrm{H}}}^{2} \leq\left\|w^{0}\right\|_{0, \Omega_{\mathrm{H}}}^{2}+\chi_{\mathrm{m}}\left\|V_{\mathrm{m}}^{0}\right\|_{0, \Omega_{\mathrm{H}}}^{2}+\tau\left\|\boldsymbol{\sigma}_{\mathrm{i}}^{\frac{1}{2}} \nabla u_{\mathrm{e}}^{0}\right\|_{0, \Omega_{\mathrm{H}}}^{2} \\
+\sum_{m=0}^{n-1} \tau\left\|I_{\mathrm{app}}^{m+1}\right\|_{0, \Omega_{\mathrm{H}}}^{2}+\alpha \sum_{m=0}^{n-1} \tau\left\|V_{\mathrm{m}}^{m+1}\right\|_{0, \Omega_{\mathrm{H}}}^{2}+\beta \sum_{m=0}^{n-1} \tau\left\|w^{m+1}\right\|_{0, \Omega_{\mathrm{H}}}^{2} .
\end{gathered}
$$

Estimate (3.26) then follows by applying Gronwall's lemma (see e.g. [24, Lemma 5.1]) under condition (3.24).

Finally, let consider the Jacobi like decoupling $\left(u_{\mathrm{e}}^{\star}, V_{\mathrm{m}}^{\star}\right)=\left(u_{\mathrm{e}}^{n}, V_{\mathrm{m}}^{n}\right)$. In this case, estimate $\mathrm{A} .43$ becomes

$$
\begin{aligned}
& \frac{1}{2}\left(\left\|w^{n+1}\right\|_{0, \Omega_{\mathrm{H}}}^{2}-\left\|w^{n}\right\|_{0, \Omega_{\mathrm{H}}}^{2}\right)+\frac{\chi_{\mathrm{m}}}{2}\left(\left\|V_{\mathrm{m}}^{n+1}\right\|_{0, \Omega_{\mathrm{H}}}^{2}-\left\|V_{\mathrm{m}}^{n}\right\|_{0, \Omega_{\mathrm{H}}}^{2}\right)+\tau\left\|\boldsymbol{\sigma}_{\mathrm{e}}^{\frac{1}{2}} \nabla u_{\mathrm{e}}^{n+1}\right\|_{0, \Omega_{\mathrm{H}}}^{2} \\
& +\tau\left\|\boldsymbol{\sigma}_{\mathrm{i}}^{\frac{1}{2}} \nabla\left(V_{\mathrm{m}}^{n+1}+u_{\mathrm{e}}^{n+1}\right)\right\|_{0, \Omega_{\mathrm{H}}}^{2}+I_{1}+\underbrace{\tau \underbrace{}_{\Omega_{\mathrm{H}}} \boldsymbol{\sigma}_{\mathrm{i}} \boldsymbol{\nabla}\left(V_{\mathrm{m}}^{n}-V_{\mathrm{m}}^{n+1}\right) \cdot \nabla u_{\mathrm{e}}^{n+1} \mathrm{~d} \boldsymbol{x}}_{I_{3}} \leq I_{2} .
\end{aligned}
$$

The new term $I_{3}$ can be bounded similarly to $I_{1}$, that is,

$$
\begin{aligned}
I_{3}= & \tau \int_{\Omega_{\mathrm{H}}} \boldsymbol{\sigma}_{\mathrm{i}} \boldsymbol{\nabla}\left(V_{\mathrm{m}}^{n}-V_{\mathrm{m}}^{n+1}\right) \cdot \boldsymbol{\nabla}\left(V_{\mathrm{m}}^{n+1}+u_{\mathrm{e}}^{n+1}\right) \mathrm{d} \boldsymbol{x}+\int_{\Omega_{\mathrm{H}}} \boldsymbol{\sigma}_{\mathrm{i}} \boldsymbol{\nabla}\left(V_{\mathrm{m}}^{n+1}-V_{\mathrm{m}}^{n}\right) \cdot \boldsymbol{\nabla} V_{\mathrm{m}}^{n+1} \mathrm{~d} \boldsymbol{x} \\
\geq & -\frac{\tau}{2}\left\|\boldsymbol{\sigma}_{\mathrm{i}}^{\frac{1}{2}} \nabla\left(V_{\mathrm{m}}^{n}-V_{\mathrm{m}}^{n+1}\right)\right\|_{0, \Omega_{\mathrm{H}}}^{2}-\frac{\tau}{2}\left\|\boldsymbol{\sigma}_{\mathrm{i}}^{\frac{1}{2}} \nabla\left(V_{\mathrm{m}}^{n+1}+u_{\mathrm{e}}^{n+1}\right)\right\|_{0, \Omega_{\mathrm{H}}}^{2} \\
& +\frac{\tau}{2}\left(\left\|\boldsymbol{\sigma}_{\mathrm{i}}^{\frac{1}{2}} \nabla V_{\mathrm{m}}^{n+1}\right\|_{0, \Omega_{\mathrm{H}}}^{2}-\left\|\boldsymbol{\sigma}_{\mathrm{i}}^{\frac{1}{2}} \nabla V_{\mathrm{m}}^{n}\right\|_{0, \Omega_{\mathrm{H}}}^{2}\right)+\frac{\tau}{2}\left\|\boldsymbol{\sigma}_{\mathrm{i}}^{\frac{1}{2}} \nabla\left(V_{\mathrm{m}}^{n+1}-V_{\mathrm{m}}^{n}\right)\right\|_{0, \Omega_{\mathrm{H}}}^{2} \\
= & \frac{\tau}{2}\left(\left\|\boldsymbol{\sigma}_{\mathrm{i}}^{\frac{1}{2}} \nabla V_{\mathrm{m}}^{n+1}\right\|_{0, \Omega_{\mathrm{H}}}^{2}-\left\|\boldsymbol{\sigma}_{\mathrm{i}}^{\frac{1}{2}} \nabla V_{\mathrm{m}}^{n}\right\|_{0, \Omega_{\mathrm{H}}}^{2}\right)-\frac{\tau}{2}\left\|\boldsymbol{\sigma}_{\mathrm{i}}^{\frac{1}{2}} \nabla\left(V_{\mathrm{m}}^{n+1}+u_{\mathrm{e}}^{n+1}\right)\right\|_{0, \Omega_{\mathrm{H}}}^{2} .
\end{aligned}
$$

Therefore, by inserting (A.44), A.45 and A.47) into (A.46), there follows that

$$
\begin{gathered}
\frac{1}{2}\left(\left\|w^{n+1}\right\|_{0, \Omega_{\mathrm{H}}}^{2}-\left\|w^{n}\right\|_{0, \Omega_{\mathrm{H}}}^{2}\right)+\frac{\chi_{\mathrm{m}}}{2}\left(\left\|V_{\mathrm{m}}^{n+1}\right\|_{0, \Omega_{\mathrm{H}}}^{2}-\left\|V_{\mathrm{m}}^{n}\right\|_{0, \Omega_{\mathrm{H}}}^{2}\right)+\tau\left\|\boldsymbol{\sigma}_{\mathrm{e}}^{\frac{1}{2}} \nabla u_{\mathrm{e}}^{n+1}\right\|_{0, \Omega_{\mathrm{H}}}^{2} \\
\frac{\tau}{2}\left(\left\|\boldsymbol{\sigma}_{\mathrm{i}}^{\frac{1}{2}} \nabla V_{\mathrm{m}}^{n+1}\right\|_{0, \Omega_{\mathrm{H}}}^{2}-\right. \\
\left.\quad\left\|\boldsymbol{\sigma}_{\mathrm{i}}^{\frac{1}{2}} \nabla V_{\mathrm{m}}^{n}\right\|_{0, \Omega_{\mathrm{H}}}^{2}\right)+\frac{\tau}{2}\left(\left\|\boldsymbol{\sigma}_{\mathrm{i}}^{\frac{1}{2}} \nabla u_{\mathrm{e}}^{n+1}\right\|_{0, \Omega_{\mathrm{H}}}^{2}-\left\|\boldsymbol{\sigma}_{\mathrm{i}}^{\frac{1}{2}} \nabla u_{\mathrm{e}}^{n}\right\|_{0, \Omega_{\mathrm{H}}}^{2}\right) \\
\leq \frac{\tau}{2}\left\|I_{\mathrm{app}}^{n+1}\right\|_{0, \Omega_{\mathrm{H}}}^{2}+\frac{\tau \alpha}{2}\left\|V_{\mathrm{m}}^{n+1}\right\|_{0, \Omega_{\mathrm{H}}}^{2}+\frac{\tau \beta}{2}\left\|w^{n+1}\right\|_{0, \Omega_{\mathrm{H}}}^{2} .
\end{gathered}
$$


Estimate (3.27) then holds, under condition (3.24), by replacing index $n$ by $m$, summing over $0 \leq m \leq n-1$ and applying Gronwall's lemma. This completes the proof.

\section{A.2 Proof of Theorem 4.4}

Since the stability of the heart-torso coupling $4.39-4.40$ does not depend on the choice of $u_{\mathrm{e}}^{\star}$ and $V_{\mathrm{m}}^{\star}$, we restrict ourselves to the case $\left(u_{\mathrm{e}}^{\star}, V_{\mathrm{m}}^{\star}\right)=\left(u_{\mathrm{e}}^{n}, V_{\mathrm{m}}^{n}\right)$. The rest of estimates can be derived in a similar fashion.

By testing (3.20)-(3.22) with $(\xi, \phi, \psi, \zeta)=\tau\left(w^{n+1}, V_{\mathrm{m}}^{n+1}, u_{\mathrm{e}}^{n+1}, u_{\mathrm{T}}^{n+1}\right)$ and after summation of the resulting expressions we have:

$$
\begin{aligned}
& \quad \frac{1}{2}\left(\left\|w^{n+1}\right\|_{0, \Omega_{\mathrm{H}}}^{2}-\left\|w^{n}\right\|_{0, \Omega_{\mathrm{H}}}^{2}\right)+\frac{\chi_{\mathrm{m}}}{2}\left(\left\|V_{\mathrm{m}}^{n+1}\right\|_{0, \Omega_{\mathrm{H}}}^{2}-\left\|V_{\mathrm{m}}^{n}\right\|_{0, \Omega_{\mathrm{H}}}^{2}\right)+\tau\left\|\boldsymbol{\sigma}_{\mathrm{e}}^{\frac{1}{2}} \boldsymbol{\nabla} u_{\mathrm{e}}^{n+1}\right\|_{0, \Omega_{\mathrm{H}}}^{2} \\
& +\tau\left\|\boldsymbol{\sigma}_{\mathrm{i}}^{\frac{1}{2}} \nabla\left(V_{\mathrm{m}}^{n+1}+u_{\mathrm{e}}^{n+1}\right)\right\|_{0, \Omega_{\mathrm{H}}}^{2}+\tau\left\|\boldsymbol{\sigma}_{\mathrm{T}}^{\frac{1}{2}} \boldsymbol{\nabla} u_{\mathrm{T}}^{n+1}\right\|_{0, \Omega_{\mathrm{T}}}^{2}+\frac{\tau \gamma \sigma_{\mathrm{T}}^{\mathrm{t}}}{h}\left\|u_{\mathrm{T}}^{n+1}-u_{\mathrm{e}}^{n+1}\right\|_{0, \Sigma}^{2} \\
& +\underbrace{\frac{\tau \gamma \sigma_{\mathrm{T}}^{\mathrm{t}}}{h} \int_{\Sigma}\left(u_{\mathrm{T}}^{n+1}-u_{\mathrm{T}}^{n}\right) u_{\mathrm{e}}^{n+1} \mathrm{~d} \boldsymbol{s}}_{I_{4}}-\underbrace{\tau \int_{\Sigma} \boldsymbol{\sigma}_{\mathrm{T}} \boldsymbol{\nabla} u_{\mathrm{T}}^{n} \cdot \boldsymbol{n}_{\mathrm{T}}\left(u_{\mathrm{T}}^{n+1}-u_{\mathrm{e}}^{n+1}\right) \mathrm{d} \boldsymbol{s}}_{I_{5}}+I_{1}+I_{3} \leq I_{2} .
\end{aligned}
$$

Terms $I_{1}, I_{2}$ and $I_{3}$ have already been estimated in the proof of Theorem 3.2 so we only need to provide bounds for $I_{4}$ and $I_{5}$.

Term $I_{4}$ is treated as follows (see [1, 10]),

$$
\begin{aligned}
I_{4}= & \frac{\tau \gamma \sigma_{\mathrm{T}}^{\mathrm{t}}}{h} \int_{\Sigma}\left(u_{\mathrm{T}}^{n+1}-u_{\mathrm{T}}^{n}\right)\left(u_{\mathrm{e}}^{n+1}-u_{\mathrm{T}}^{n+1}\right) \mathrm{d} \boldsymbol{s}+\frac{\tau \gamma \sigma_{\mathrm{T}}^{\mathrm{t}}}{h} \int_{\Sigma}\left(u_{\mathrm{T}}^{n+1}-u_{\mathrm{T}}^{n}\right) u_{\mathrm{T}}^{n+1} \mathrm{~d} \boldsymbol{s} \\
\geq & -\frac{\tau \gamma \sigma_{\mathrm{T}}^{\mathrm{t}}}{2 h}\left\|u_{\mathrm{T}}^{n+1}-u_{\mathrm{T}}^{n}\right\|_{0, \Sigma}^{2}-\frac{\tau \gamma \sigma_{\mathrm{T}}^{\mathrm{t}}}{2 h}\left\|u_{\mathrm{e}}^{n+1}-u_{\mathrm{T}}^{n+1}\right\|_{0, \Sigma}^{2} \\
& +\frac{\tau \gamma \sigma_{\mathrm{T}}^{\mathrm{t}}}{2 h}\left(\left\|u_{\mathrm{T}}^{n+1}\right\|_{0, \Sigma}^{2}-\left\|u_{\mathrm{T}}^{n}\right\|_{0, \Sigma}^{2}\right)+\frac{\tau \gamma \sigma_{\mathrm{T}}^{\mathrm{t}}}{2 h}\left\|u_{\mathrm{T}}^{n+1}-u_{\mathrm{T}}^{n}\right\|_{0, \Sigma}^{2} \\
= & \frac{\tau \gamma \sigma_{\mathrm{T}}^{\mathrm{t}}}{2 h}\left(\left\|u_{\mathrm{T}}^{n+1}\right\|_{0, \Sigma}^{2}-\left\|u_{\mathrm{T}}^{n}\right\|_{0, \Sigma}^{2}\right)-\frac{\tau \gamma \sigma_{\mathrm{T}}^{\mathrm{t}}}{2 h}\left\|u_{\mathrm{e}}^{n+1}-u_{\mathrm{T}}^{n+1}\right\|_{0, \Sigma}^{2} .
\end{aligned}
$$

On the other hand, using 4.30 , for the last term we have

$$
\begin{aligned}
I_{5} & \geq-\frac{\tau h}{\gamma}\left\|\left(\sigma_{\mathrm{T}}^{\mathrm{t}}\right)^{\frac{1}{2}} \nabla u_{\mathrm{T}}^{n}\right\|_{0, \Sigma}^{2}-\frac{\tau \gamma \sigma_{\mathrm{T}}^{\mathrm{t}}}{4 h}\left\|u_{\mathrm{e}}^{n+1}-u_{\mathrm{T}}^{n+1}\right\|_{0, \Sigma}^{2} \\
& \geq-\frac{\tau C_{\mathrm{ti}}}{\gamma}\left\|\sigma_{\mathrm{T}}^{\frac{1}{2}} \nabla u_{\mathrm{T}}^{n}\right\|_{0, \Omega_{\mathrm{T}}}^{2}-\frac{\tau \gamma \sigma_{\mathrm{T}}^{\mathrm{t}}}{4 h}\left\|u_{\mathrm{e}}^{n+1}-u_{\mathrm{T}}^{n+1}\right\|_{0, \Sigma}^{2}
\end{aligned}
$$


Therefore, by inserting A.44, A.45, A.47, A.49 and A.50 into A.48 we get the estimate

$$
\begin{gathered}
\frac{1}{2}\left(\left\|w^{n+1}\right\|_{0, \Omega_{\mathrm{H}}}^{2}-\left\|w^{n}\right\|_{0, \Omega_{\mathrm{H}}}^{2}\right)+\frac{\chi_{\mathrm{m}}}{2}\left(\left\|V_{\mathrm{m}}^{n+1}\right\|_{0, \Omega_{\mathrm{H}}}^{2}-\left\|V_{\mathrm{m}}^{n}\right\|_{0, \Omega_{\mathrm{H}}}^{2}\right)+\tau\left\|\boldsymbol{\sigma}_{\mathrm{e}}^{\frac{1}{2}} \nabla u_{\mathrm{e}}^{n+1}\right\|_{0, \Omega_{\mathrm{H}}}^{2} \\
+\tau\left(\left\|\boldsymbol{\sigma}_{\mathrm{T}}^{\frac{1}{2}} \nabla u_{\mathrm{T}}^{n+1}\right\|_{0, \Omega_{\mathrm{T}}}^{2}-\frac{C_{\mathrm{ti}}}{\gamma}\left\|\boldsymbol{\sigma}_{\mathrm{T}}^{\frac{1}{2}} \nabla u_{\mathrm{T}}^{n}\right\|_{0, \Omega_{\mathrm{T}}}^{2}\right)+\frac{\tau \gamma \sigma_{\mathrm{T}}^{\mathrm{t}}}{4 h}\left\|u_{\mathrm{T}}^{n+1}-u_{\mathrm{e}}^{n+1}\right\|_{0, \Sigma}^{2} \\
+\frac{\tau \gamma \sigma_{\mathrm{T}}^{\mathrm{t}}}{2 h}\left(\left\|u_{\mathrm{T}}^{n+1}\right\|_{0, \Sigma}^{2}-\left\|u_{\mathrm{T}}^{n}\right\|_{0, \Sigma}^{2}\right)+\frac{\tau}{2}\left(\left\|\boldsymbol{\sigma}_{\mathrm{i}}^{\frac{1}{2}} \nabla V_{\mathrm{m}}^{n+1}\right\|_{0, \Omega_{\mathrm{H}}}^{2}-\left\|\boldsymbol{\sigma}_{\mathrm{i}}^{\frac{1}{2}} \nabla V_{\mathrm{m}}^{n}\right\|_{0, \Omega_{\mathrm{H}}}^{2}\right) \\
+\frac{\tau}{2}\left(\left\|\boldsymbol{\sigma}_{\mathrm{i}}^{\frac{1}{2}} \nabla u_{\mathrm{e}}^{n+1}\right\|_{0, \Omega_{\mathrm{H}}}^{2}-\left\|\boldsymbol{\sigma}_{\mathrm{i}}^{\frac{1}{2}} \nabla u_{\mathrm{e}}^{n}\right\|_{0, \Omega_{\mathrm{H}}}^{2}\right) \leq \frac{\tau}{2}\left\|I_{\mathrm{app}}^{n+1}\right\|_{0, \Omega_{\mathrm{H}}}^{2} \\
+\frac{\tau \alpha}{2}\left\|V_{\mathrm{m}}^{n+1}\right\|_{0, \Omega_{\mathrm{H}}}^{2}+\frac{\tau \beta}{2}\left\|w^{n+1}\right\|_{0, \Omega_{\mathrm{H}}}^{2} .
\end{gathered}
$$

Estimate 4.42 then follows, under conditions 4.41 and 3.24, by replacing index $n$ by $m$, summing over $0 \leq m \leq n-1$ and applying Gronwall's lemma, which completes the proof.

\section{Acknowledgements}

This work was partially supported by INRIA through its large scope initiative CardioSense3D (www.inria.fr/CardioSense3D). We wish to thank JeanFrédéric Gerbeau and Muriel Boulakia for many fruitful discussions on ECG modeling and simulation. We also thank Philippe Moireau and Elsie Phé for their work on the anatomical models and meshes.

\section{References}

[1] M. Astorino, F. Chouly, and M.A. Fernández. Robin based semi-implicit coupling in fluid-structure interaction: Stability analysis and numerics. SIAM J. Sci. Comput., 31(6):4041-4065, 2009.

[2] T.M. Austin, M.L. Trew, and A.J. Pullan. Solving the cardiac bidomain equations for discontinuous conductivities. IEEE Trans. Biomed. Eng., 53(7):1265-72, 2006.

[3] M. Bendahmane and K.H. Karlsen. Analysis of a class of degenerate reaction-diffusion systems and the bidomain model of cardiac tissue. Netw. Heterog. Media, 1(1):185-218, 2006.

[4] M. Boulakia, S. Cazeau, M.A. Fernández, J.-F. Gerbeau, and N. Zemzemi. Mathematical Modeling of Electrocardiograms: A Numerical Study. Ann. Biomed. Eng., 38(3):1071-1097, 2010.

[5] M. Boulakia, M.A. Fernández, J.-F. Gerbeau, and N. Zemzemi. Towards the numerical simulation of electrocardiograms. In F.B. Sachse and G. Seemann, editors, Functional Imaging and Modeling of the Heart, number 4466 in Lecture Notes in Computer Science, pages 240-249. Springer-Verlag, 2007. 
[6] M. Boulakia, M.A. Fernández, J.-F. Gerbeau, and N. Zemzemi. A coupled system of PDEs and ODEs arising in electrocardiograms modelling. Applied Math. Res. Exp., 2008(abn002):28, 2008.

[7] Y. Bourgault, Y. Coudière, and C. Pierre. Existence and uniqeness of the solution for the bidomain model used in cardiac electrophysiology. Nonlinear Anal. Real World Appl., 10(1):458-482, 2009.

[8] Y. Bourgault, M. Ethier, and V.G. Le Blanc. Simulation of electrophysiological waves with an unstructured finite element method. M2AN Math. Model. Numer. Anal., 37(4):649-661, 2003.

[9] M. Buist and A. Pullan. Torso coupling techniques for the forward problem of electrocardiography. Ann. Biomed. Eng., 30(10):1299-1312, 2002.

[10] E. Burman and M.A. Fernández. Stabilization of explicit coupling in fluidstructure interaction involving fluid incompressibility. Comput. Methods Appl. Mech. Engrg., 198(5-8):766-784, 2009.

[11] D. Chapelle, M.A. Fernández, J.-F. Gerbeau, P. Moireau, J. Sainte-Maire, and N. Zemzemi. Numerical simulation of the electromechanical activity of the heart. In N. Ayache, H. Delingette, and M. Sermesant, editors, Functional Imaging and Modeling of the Heart, volume 5528 of Lecture Notes in Computer Science, pages 357-365. Springer-Verlag, 2009.

[12] D. Chapelle, M.A. Fernández, J.-F. Gerbeau, P. Moireau, and N. Zemzemi. A 3D model for the electromechanical activity of the heart. Submitted, 2009.

[13] J. Clements, J. Nenonen, P.K.J. Li, and B.M. Horacek. Activation dynamics in anisotropic cardiac tissue via decoupling. Annals of Biomedical Engineering, 32(7):984-990, 2004.

[14] P. Colli Franzone and L.F. Pavarino. A parallel solver for reaction-diffusion systems in computational electrocardiology. Math. Models Methods Appl. Sci., 14(6):883-911, 2004.

[15] P. Colli Franzone, L.F. Pavarino, and B. Taccardi. Simulating patterns of excitation, repolarization and action potential duration with cardiac bidomain and monodomain models. Math. Biosci., 197(1):35-66, 2005.

[16] P. Colli Franzone and G. Savaré. Degenerate evolution systems modeling the cardiac electric field at micro- and macroscopic level. In Evolution equations, semigroups and functional analysis (Milano, 2000), volume 50 of Progr. Nonlinear Differential Equations Appl., pages 49-78. Birkhäuser, Basel, 2002.

[17] I.R. Efimov, R.A. Gray, and B.J. Roth. Virtual electrodes and deexcitation: new insights into fibrillation induction and defibrillation. J. Cardiovasc. Electrophysiol., 11(3):339-353, 2000.

[18] A. Ern and J.-L. Guermond. Theory and practice of finite elements, volume 159 of Applied Mathematical Sciences. Springer-Verlag, New York, 2004. 
[19] M. Ethier and Y. Bourgault. Semi-Implicit Time-Discretization Schemes for the Bidomain Model. SIAM J. Numer. Anal., 46:2443, 2008.

[20] R. Fitzhugh. Impulses and physiological states in theoretical models of nerve membrane. Biophys. J., 1:445-465, 1961.

[21] P. Frey. Yams: A fully automatic adaptive isotropic surface remeshing procedure. Technical Report RT-0252, INRIA, Rocquencourt, France, 2001.

[22] P.L. George, F. Hecht, and E. Saltel. Fully automatic mesh generator for 3d domains of any shape. Impact of Comp. in Sci. ans Eng.,, 2:187-218, 1990 .

[23] R.M. Gulrajani. Models of the electrical activity of the heart and computer simulation of the electrocardiogram. Crit. Rev. Biomed. Eng., 16(1):1-6, 1988.

[24] J.G. Heywood and R. Rannacher. Finite-element approximation of the nonstationary Navier-Stokes problem. IV. Error analysis for second-order time discretization. SIAM J. Numer. Anal., 27(2):353-384, 1990.

[25] N. Hooke, C.S. Henriquez, P. Lanzkron, and D. Rose. Linear algebraic transformations of the bidomain equations: implications for numerical methods. Math. Biosci., 120(2):127-145, 1994.

[26] G. Huiskamp. Simulation of depolarization in a membrane-equations-based model of the anisotropic ventricle. IEEE Trans. Biomed. Eng., 5045(7):847$855,1998$.

[27] J. P. Keener and K. Bogar. A numerical method for the solution of the bidomain equations in cardiac tissue. Chaos, 8(1):234-241, 1998.

[28] W. Krassowska and J.C. Neu. Effective boundary conditions for syncitial tissues. IEEE Trans. Biomed. Eng., 41(2):143-150, 1994.

[29] L.J. Leon and B.M. Horácek. Computer model of excitation and recovery in the anisotropic myocardium. I. Rectangular and cubic arrays of excitable elements. J. Electrocardiol., 24(1):1-15, 1991.

[30] G. T. Lines, M. L. Buist, P. Grottum, A. J. Pullan, J. Sundnes, and A. Tveito. Mathematical models and numerical methods for the forward problem in cardiac electrophysiology. Comput. Visual. Sci., 5(4):215-239, 2003.

[31] G.T. Lines, P. Grøttum, and A. Tveito. Modeling the electrical activity of the heart: a bidomain model of the ventricles embedded in a torso. Comput. Vis. Sci., 5(4):195-213, 2003.

[32] S. Linge, J. Sundnes, M. Hanslien, G.T. Lines, and A. Tveito. Numerical solution of the bidomain equations. Philos. Transact. A. Math. Phys. Eng. Sci., 367(1895):1931-1950, 2009.

[33] C.C. Mitchell and D.G. Schaeffer. A two-current model for the dynamics of cardiac membrane. Bulletin Math. Bio., 65:767-793, 2003. 
[34] M. Murillo and X.-C. Cai. A fully implicit parallel algorithm for simulating the non-linear electrical activity of the heart. Numer. Linear Algebra Appl., 11(2-3):261-277, 2004.

[35] J.S. Nagumo, S. Arimoto, and S. Yoshizawa. An active pulse transmission line stimulating nerve axon. Proc. IRE, (50):2061-2071, 1962.

[36] M. Pennacchio and V. Simoncini. Efficient algebraic solution of reactiondiffusion systems for the cardiac excitation process. J. Comput. Appl. Math., 145(1):49-70, 2002.

[37] M. Potse, B. Dubé, and M. Gulrajani. ECG simulations with realistic human membrane, heart, and torso models. In Proceedings of the 25th Annual Intemational Conference of the IEEE EMBS, pages 70-73, 2003.

[38] M. Potse, B. Dube, J. Richer, A. Vinet, and R. M. Gulrajani. A comparison of monodomain and bidomain reaction-diffusion models for action potential propagation in the human heart. IEEE Trans. Biomed. Eng., 53(12):2425$2435,2006$.

[39] M. Potse, B. Dubé, and A. Vinet. Cardiac anisotropy in boundary-element models for the electrocardiogram. Med. Biol. Eng. Comput., 47:719-729, 2009.

[40] A.J. Pullan, M.L. Buist, and L.K. Cheng. Mathematically modelling the electrical activity of the heart: From cell to body surface and back again. World Scientific Publishing Co. Pte. Ltd., Hackensack, NJ, 2005.

[41] N. Skouibine, K. Trayanova and P. Moore. A numerically efficient model for simulation of defibrillation in an active bidomain sheet of myocardium. Math. Biosci., 166(1):85-100, 2000.

[42] R.A. Stephenson. On the uniqueness of the square-root of a symmetric, positive-definite tensor. J. Elasticity, 10(2):213-214, 1980.

[43] H.J. Stetter. The defect correction principle and discretization methods. Numer. Math., 29:425-443, 1978.

[44] J. Sundnes, G.T. Lines, X. Cai, B.F. Nielsen, K.-A. Mardal, and A. Tveito. Computing the electrical activity in the heart. Springer-Verlag, 2006.

[45] J. Sundnes, G.T. Lines, and A. Tveito. Efficient solution of ordinary differential equations modeling electrical activity in cardiac cells. Math. Biosci., 172(2):55-72, 2001.

[46] J. Sundnes, G.T. Lines, and A. Tveito. An operator splitting method for solving the bidomain equations coupled to a volume conductor model for the torso. Math. Biosci., 194(2):233-248, 2005.

[47] V. Thomée. Galerkin finite element methods for parabolic problems, volume 25 of Springer Series in Computational Mathematics. Springer-Verlag, Berlin, second edition, 2006.

[48] L. Tung. A bi-domain model for describing ischemic myocardial $D-C$ potentials. PhD thesis, MIT, 1978. 
[49] M. Veneroni. Reaction-diffusion systems for the macroscopic bidomain model of the cardiac electric field. Nonlinear Anal. Real World Appl., 10(2):849-868, 2009.

[50] E.J. Vigmond, R. Weber dos Santos, A.J. Prassl, M Deo, and G. Plank. Solvers for the cardiac bidomain equations. Progr. Biophys. Molec. Biol., 96(1-3):3-18, 2008. 


\section{Contents}

1 Introduction 3

2 Mathematical models 4

2.1 Isolated heart . . . . . . . . . . . . . . . . . . . . 4

2.2 Coupling with torso: ECG modeling . . . . . . . . . . . . . 6

\begin{tabular}{|lll}
3 & Decoupled time-marching for the bidomain equation & 7
\end{tabular}

3.1 Preliminaries ................... 8

3.2 Time semi-discrete formulations: decoupled time-marching schemes 8

3.3 Stability analysis . . . . . . . . . . . . . 9

4 Decoupled time-marching for ECG numerical simulation 11

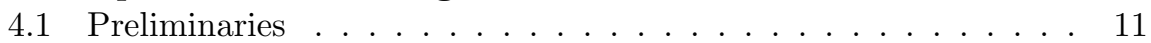

$4.2 \quad$ Fully discrete formulation: decoupled time-marching schemes . . 13

4.3 Stability analysis . . . . . . . . . . . . . . . . 14

5 Numerical results 15

5.1 Mono-dimensional study . . . . . . . . . . . . . . . . . . . 15

5.1 .1 Simulation data $. \ldots \ldots . . \ldots . . \ldots 15$

5.1 .2 Isolated heart . . . . . . . . . . . . . . . . . . . . 16

$5.1 .3 \quad$ Heart-torso coupling . . . . . . . . . . . . . . 16

5.2 Three-dimensional study . . . . . . . . . . . . . . . . . . 18

5.2 .1 Simulation data . . . . . . . . . . . . . . . . 18

5.2 .2 Isolated heart . . . . . . . . . . . . . . . . . . 20

5.2 .3 12-lead ECG . . . . . . . . . . . . . . . . . 20



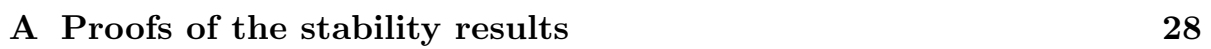

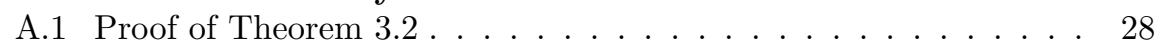

A.2 Proof of Theorem $4.4 \ldots \ldots \ldots$. . . . . . . . . . . . 32 
Unité de recherche INRIA Rocquencourt Domaine de Voluceau - Rocquencourt - BP 105 - 78153 Le Chesnay Cedex (France)

Unité de recherche INRIA Futurs : Parc Club Orsay Université - ZAC des Vignes 4, rue Jacques Monod - 91893 ORSAY Cedex (France)

Unité de recherche INRIA Lorraine : LORIA, Technopôle de Nancy-Brabois - Campus scientifique 615, rue du Jardin Botanique - BP 101 - 54602 Villers-lès-Nancy Cedex (France)

Unité de recherche INRIA Rennes : IRISA, Campus universitaire de Beaulieu - 35042 Rennes Cedex (France)

Unité de recherche INRIA Rhône-Alpes : 655, avenue de l'Europe - 38334 Montbonnot Saint-Ismier (France) Unité de recherche INRIA Sophia Antipolis : 2004, route des Lucioles - BP 93 - 06902 Sophia Antipolis Cedex (France) 